\title{
Exact quantification by the nonparametric fast Padé transform using only shape estimation of high-order derivatives of envelopes
}

\author{
Dževad Belkić1,2 . Karen Belkić ${ }^{1,2,3,4}$
}

Received: 23 October 2017 / Accepted: 16 November 2017 / Published online: 2 December 2017 (C) The Author(s) 2017. This article is an open access publication

\begin{abstract}
This study deals exclusively with total shape spectra (envelopes). Yet, the goal is to exactly reconstruct all the components of the given envelope by relying only upon non-parametric signal processors (shape estimators). To this end, the socalled derivative envelope spectra are investigated. A derivative spectrum is the result of the application of the differentiation transform $(\mathrm{d} / \mathrm{d} \nu)^{m}$ to the given conventional spectrum. Here, non-negative integer $m$ is the order of differentiation and $v$ is the real linear sweep frequency. For the customary envelope (the zeroth-order derivative, $m=0$ ), we use the non-parametric fast Padé transform to generate the derivative fast Padé transform (dFPT). Explicit computations are carried out by successively increasing the differentiation order $m$ from low through intermediate to high values of derivatives of complex envelopes. The dFPT can disentangle the spectrally crowded regions by splitting apart any multiplet of closely packed peaks. Hidden resonances, even those that are very weak, can be not only visualized, but also exactly quantified by the dFPT, despite performing shape estimations alone. Most importantly, while the envelopes in the derivative fast Fourier transform exhibit huge noise amplification with increasing $m$, the same-order of the differentiation transform in the dFPT acts as an effective noise suppressor. The results of the dFPT are illustrated for the envelopes with
\end{abstract}

$凶$ Dževad Belkić

Dzevad.Belkic@ki.se

1 Department of Oncology-Pathology, Karolinska Institute, Building P-9, 2nd floor, P.O. Box 260, 17176 Stockholm, Sweden

2 Medical Radiation Physics and Nuclear Medicine, Karolinska University Hospital, Stockholm, Sweden

3 School of Community and Global Health, Claremont Graduate University, Claremont, CA, USA

4 Institute for Prevention Research, Keck School of Medicine, University of Southern California, Alhambra, CA, USA 
overlapping peaks stemming from synthesized noise-free and noise-contaminated time signals associated with encoding by in vitro proton magnetic resonance spectroscopy (MRS) of breast cancer tissue. This new methodology is anticipated to significantly enhance resolution as well as signal-to-noise ratio and the overall performance of single-voxel MRS in clinical diagnostics. It is also expected to be of special benefit for volumetric coverage of the scanned tissue by magnetic resonance spectroscopic imaging.

Keywords Magnetic resonance spectroscopy - Breast cancer diagnostics · Mathematical optimization · Fast Padé transform - Derivative spectra

\section{Abbreviations}

\begin{tabular}{|c|c|}
\hline Ala & Alanine \\
\hline AR & Auto-regression \\
\hline ARMA & Auto-regressive moving average \\
\hline $\mathrm{au}$ & Arbitrary units \\
\hline$\beta$-Glc & $\beta$-glucose \\
\hline $\mathrm{BW}$ & Bandwidth \\
\hline Cho & Choline \\
\hline dDFT & Derivative discrete Fourier transform \\
\hline DFT & Discrete Fourier transform \\
\hline $\mathrm{dFFT}$ & Derivative fast Fourier transform \\
\hline dFPT & Derivative fast Padé transform \\
\hline dMRS & Derivative magnetic resonance spectroscopy \\
\hline dMRSI & Derivative magnetic resonance spectroscopic imaging \\
\hline FFT & Fast Fourier transform \\
\hline FID & Free induction decay \\
\hline FPT & Fast Padé transform \\
\hline FWHM & Full-widths at half-maximum \\
\hline GPC & Glycerophosphocholine \\
\hline $\mathrm{Hz}$ & Hertz \\
\hline Lac & Lactate \\
\hline MA & Moving average \\
\hline MR & Magnetic resonance \\
\hline MRI & Magnetic resonance imaging \\
\hline MRS & Magnetic resonance spectroscopy \\
\hline MRSI & Magnetic resonance spectroscopic imaging \\
\hline m-Ins & Myoinositol \\
\hline NMR & Nuclear magnetic resonance \\
\hline PA & Padé approximant \\
\hline $\mathrm{PC}$ & Phosphocholine \\
\hline $\mathrm{PE}$ & Phosphoethanolamine \\
\hline ppm & Parts per million \\
\hline RMS & Root mean square \\
\hline
\end{tabular}




$\begin{array}{ll}\text { S } & \text { Second } \\ \text { SNR } & \text { Signal-to-noise ratio } \\ \text { SRI } & \text { Spectral region of interest } \\ \text { T } & \text { Tesla } \\ \text { Tau } & \text { Taurine } \\ \text { TSP } & \text { 3-(Trimethylsilyl-)3,3,2,2-tetradeutero-propionic acid } \\ \text { ww } & \text { Wet weight }\end{array}$

\section{Introduction}

In the present study, we are furthering our earlier investigations on non-parametric estimations of lineshapes from total shape spectra or envelopes. With this goal, the high-resolution signal processing method, the fast Padé transform (FPT), is used. In e.g. the diagonal form of the FPT, the frequency envelope spectrum in the complex mode is given by the quotient of two polynomials $P_{K} / Q_{K}$ of the common degree $K$. The numerator $P_{K}$ gives the spectral zeros and suppresses noise by a moving average (MA) process. The denominator $Q_{K}$ yields the spectral poles, and the expansion coefficients of this polynomial coincide with those of an auto-regressive (AR) process. The combination of AR and MA processes yields the method known as the auto-regressive moving average (ARMA) [1]. The zeros and poles of the complex envelope $P_{K} / Q_{K}$ are determined exclusively by $P_{K}$ and $Q_{K}$, respectively. This occurs because the spectrum $P_{K} / Q_{K}$ is a meromorphic function. A meromorphic function is a function whose only singularities are poles.

By its default rational polynomial representation, $P_{K} / Q_{K}$, the diagonal FPT is of a polar structure, which naturally yields the peaks as the main signature of spectral envelopes. By comparison, the fast Fourier transform (FFT), as a low-resolution processor, is a single polynomial, which has no polar structure. Critically, the FFT cannot suppress noise.

Generally, if two or more resonances overlap too closely, they would invariably appear as a single peak in an envelope. Even more misleading, such a compound resonance could be an absorptive, symmetrical Lorentzian with no hint whatsoever pointing to its constituent components. This is a very common occurrence for total shape spectra reconstructed by all signal processers. For example, the FFT as a nonparametric processor cannot visualize hidden resonances in composite peaks. The same applies to other non-parametric processors with shape estimation alone (i.e. without resorting to quantification, which is in the mathematical literature known as spectral analysis).

The exception is the non-parametric FPT, for which the situation is sharply different. In the FPT, both the AR and MA partitions can be exploited to split apart the tightly overlaid peaks and, thus, visualize the hidden component resonances, in compound spectral structures of envelopes. This observation was the motivation of our earlier studies [2,3] to introduce the so-called "partitioned envelope spectra" in the FPT. Therein, it was shown that the unique combination of the pertinent portions of the AR and MA processes produced the partitioned envelope spectra that clearly visualized the most tightly overlapped resonances. This is a very important accomplishment demon- 
strating that quickly, prior to any quantification, the partitioned envelopes from the FPT can peer into the hidden structure of total shape spectra. Such a hint could advantageously guide the subsequent local spectral analysis by focusing on the crowded regions of high spectral density. Although remarkable in itself, this achievement was, nevertheless, still in the realm of qualitative estimations. This occurred because the visualized overlapped components did not exhibit e.g. the correct peak heights, nor their correct peak height ratios. Note that the partitioned envelopes in the FFT exhibit no separation of peaks that in the non-partitioned Fourier spectra appear as overlapped resonances.

In the current work, we proceed one critical step further by inquiring as to whether it would be possible to both visualize and correctly quantify the components of compound resonances by the non-parametric FPT through solely shape estimation of spectral envelopes. The answer to this query is presently investigated by introducing the derivative fast Padé transform (dFPT). The dFPT gives the derivative envelopes by applying the differential operator $(\mathrm{d} / \mathrm{d} \nu)^{m}$ to the seed total shape spectrum $P_{K} / Q_{K}$, generated non-parametrically in the customary FPT (zeroth-order derivative, $m=0$ ). Here, $v$ is the sweep real-valued linear frequency. The dFPT, exclusively by way of shape estimations of envelopes, and without any partitioning, is examined with regard to visualization and quantification of the hidden components of perfectly symmetrical absorptive Lorentzians. This is studied for time signals and the ensuing envelope spectra typically encountered in magnetic resonance spectroscopy (MRS).

The chosen examples refer to noise-free and noise-corrupted time signals associated with in vitro proton MRS data encoded from breast cancer tissue. This illustration is selected because of its utmost clinical relevance. Therein, the prime goal of early tumor diagnostics (that, in turn, significantly enhances the chances of successful treatment and potential cure) is to reliably identify a recognized cancer biomarker, phosphocholine (PC), which is completely masked by a closely adjacent metabolite, phosphoethanolamine (PE). Namely, the PC peak is wholly dominated by the PE resonance, and their chemical shift separation is only 0.001 parts per million (ppm).

Noise-contaminated MRS data are especially demanding for separately visualizing and simultaneously quantifying overlapped resonances. This task is expected to be flagrantly failed by the derivative fast Fourier transform (dFFT), where the detrimental influence of noise would be amplified by the differentiation operator $(d / d \nu)^{m}$. In sharp contrast, the same differentiation transform $(\mathrm{d} / \mathrm{d} \nu)^{m}$ is foreseen as an efficient noise suppressor in the derivative fast Padé transform, dFPT.

Single voxels used in MRS may not be sufficiently representative of the status of the scanned tissue. That is why, whenever there is a doubt, multi-voxels are employed in scanning, yielding volumetric coverage of the examined tissue. This magnetic resonance (MR) modality is called magnetic resonance spectroscopic imaging (MRSI). It advantageously unifies both magnetic resonance imaging (MRI) and MRS to yield anatomic (morphologic) and functional (metabolic) information, as reviewed in e.g. Ref. [4]. There are, however, three "price tags" for such a combined diagnostic modality. Firstly, signal-to-noise ratio (SNR) is deteriorated in MRSI relative to MRS. Secondly, MRSI is more time consuming than MRS. It requires keeping the patient longer in the scanner during data acquisition from multiple voxels. Thirdly, analysis of the encoded data is computationally far more demanding in MRSI than in MRS 
because the former is tasked with reconstructing and interpreting thousands of spectra to extract (by quantification) the diagnostically relevant information.

It is this threefold obstacle which has hampered further exploration of MRSI in the clinical setting. Because of the obvious advantage of volumetric tissue coverage in MRSI with respect to MRS, and due to the appearance of the same type of encoded time signals in both modalities, it is important to ascertain whether there is a way to simultaneously surmount all three mentioned difficulties.

The parametric FPT has heretofore come to the rescue with its superior highresolution by way of solving the quantification problem. Moreover, the non-parametric FPT for partitioned spectral envelopes can disentangle overlapped resonances in the multiple voxels of the tissue scanned by MRSI. This partitioned-spectra-guided MRSI can substantially reduce the large dimensionality of the initial problem and set the stage for an efficient local quantification in narrower frequency ranges. Thus, to complete the extraction of spectral information, the partitioned non-parametric FPT still relies upon quantification by the subsequent parametric FPT to obtain the actual concentrations. The reason is because the partitioned non-parametric FPT itself gives only qualitative information, as it performs estimations of lineshapes of spectral envelopes alone.

In the present paper, we proceed beyond the traditionally conceived shape estimators by asking the question: is there a way of making these non-parametric processors intrinsically quantitative (by accurate reconstruction of the position, width, height and phase of each component peak of all the physical resonances without any fitting)? Specifically, we seek to find out what else could be in the store of the FPT that could rely only upon non-parametric shape estimation and yet would yield exact quantification. The answer is sought in the derivative fast Padé transform, dFPT. It is this processor which is potentially of utmost usefulness for both MRS and MRSI. Further, it is foreseen that the dFPT could increase the spectral resolution elsewhere, since the presently studied time signals are ubiquitous across interdisciplinary research fields.

\section{Theory}

\subsection{Time signals in quantum physics}

The research field of signal processing abounds with different categories of methods. The two main categories are "shape" and "parameter estimators". Traditionally, shape estimation is qualitative, as it is restricted to reconstruction of spectral lineshapes. Parameter estimation is quantitative, since it yields the peak positions, widths, heights and phases of resonances. Such parameters are important because they supply e.g. the critically important information about the abundance or concentrations of various components in a mixture of substances from the investigated matter. This topic goes under the general umbrella of versatile investigations of the structure of matter. The theoretically analyzed data, the input time signals, that offer the opportunity to peer into the structure of various forms of matter, are measured by a myriad of spectroscopies (infrared, ultraviolet, nuclear magnetic resonance, ...) and spectrometries (ion-cyclotron resonance mass spectrometry, ...). 
Time signals measured (recorded, acquired, encoded) by these modalities stem from vastly different transitions of nuclei, atoms or molecules from one to another energy level of a system in the matter under study. Such transitions result from various types of interactions of external perturbations or agents with the considered system. External perturbations are either waves (electromagnetic fields,...) or particles (electrons, ions,...) and the targeted systems are atomic nuclei, atoms, molecules, etc. In magnetic resonance spectroscopy, MRS, used e.g. in cancer diagnostics, the non-invasively studied matter is tissue (or more precisely the tissue's molecules called metabolites), and the external perturbations are electromagnetic fields (static as well as gradient magnetic fields and radiofrequency pulses).

The transitions of the system, on sub-nuclear, nuclear, atomic or molecular levels are quantized in energies, spins and other observables. These all obey the rules of quantum physics which dictates which transitions are allowed and which are forbidden. Quantum physics predicts the amplitudes of the probabilities for these transitions to occur. It also predicts the waveforms of the time signals $c(t)$ emitted as a response of the system's states to external perturbations. Likewise, quantum physics gives the spectrum $G(\omega)$, the lineshapes and the peak (or resonance) parameters (position, width, height, phase). Universally, these time signals are described by quantum mechanical auto-correlation functions through the time evolution operator (the generator of the system's dynamics) which gives:

$$
c(t)=\sum_{k=1}^{K} d_{k} \mathrm{e}^{-i \omega_{k} t} ; c(t)=0 \quad \text { for } \quad t<0, \quad \operatorname{Im}\left(\omega_{k}\right)<0
$$

where $t$ is the continuous time variable and $K$ is the finite or infinite number of components $(K=1,2, \ldots)$. The complex-valued pair $\left\{\omega_{k}, d_{k}\right\}(1 \leq k \leq K)$ represents the system's eigen-parameters, consisting of the eigen-frequencies $\left\{\omega_{k}\right\}$ and eigenamplitudes $\left\{d_{k}\right\}$. The digitalized version of (1) is obtained by discretizing $t$ according to $t=n \tau(n=0,1,2, \ldots, N-1)$, with $\tau$ being the sampling rate, so that:

$$
c_{n}=\sum_{k=1}^{K} d_{k} \mathrm{e}^{-i \omega_{k} n \tau} ;(0 \leq n \leq N-1)
$$

where $N$ is the total signal length. The total duration of the time signal (or total acquisition time) $T$ is given by $T=N \tau$.

\subsection{Frequency spectra in quantum physics}

The quantum-physics spectrum $G(\omega)$ associated with the auto-correlation function $c(t)$ with $T=\infty$ is defined at the angular frequency $\omega$ by the Green function:

$$
G(\omega)=\int_{0}^{\infty} \mathrm{d} t \mathrm{e}^{i \omega t} c(t)
$$


For $c(t)$ given by (1), the integral in (3) yields:

$$
G(\omega)=i \sum_{k=1}^{K} \frac{d_{k}}{\omega-\omega_{k}}
$$

This spectrum is a sum of $K$ components, each of which is a complex-valued Lorentzian (response function) of the form of a rational polynomial:

$$
G_{k}(\omega)=\frac{i d_{k}}{\omega-\omega_{k}} .
$$

The sum over $k$ in (4) can be explicitly carried out with the following result:

$$
G(\omega)=\frac{P_{K-1}(\omega)}{Q_{K}(\omega)} .
$$

Quantities $P_{K-1}(\omega)$ and $Q_{K}(\omega)$ are polynomials of degree $K-1$ and $K$, respectively, whose expansion coefficients are formally identifiable from (4) in terms of $\omega_{k}$ and $d_{k}$.

\subsection{Rational polynomials in signal processing}

From the mathematical viewpoint, the quantum-mechanically predicted spectrum $G(\omega)$ in (6), as a rational polynomial (a ratio of two polynomials), is recognized as the para-diagonal Padé approximant (PA). In signal processing, the PA is known as the fast Padé transform, FPT [1,5]. This leaves little doubt as to which of the existing signal processors is most suitable for spectral analysis. Conversely, the spectrum $G(\omega)$ in (4), derived by integration (3) of the time signal $c(t)$ from (1), can alternatively be generated through decomposition of the Padé polynomial quotient $P_{K-1}(\omega) / Q_{K}(\omega)$ from (6) into its Heaviside partial fractions. Note that both the $k$ th component $G_{k}(\omega)$ in (5) and the total shape spectrum $G(\omega)$ in (6) are the para-diagonal Padé approximants. In practice, $P_{K-1}(\omega)$ and $Q_{K}(\omega)$ in (6) are, of course, not deduced from (4). Rather, it is the other way around. First, $P_{K-1}(\omega)$ and $Q_{K}(\omega)$ are determined. Then, the partial fractions are made for $P_{K-1}(\omega) / Q_{K}(\omega)$ to give the Heaviside representation (4).

The FPT provides both shape and parameter estimations. For a given power series, with signal points $\left\{c_{n}\right\}(n=0,1,2, \ldots)$ as the expansion coefficients, Padé-based estimation of spectral lineshapes is carried out by computing, e.g. the general quotients $P_{L}(\omega) / Q_{K}(\omega)$ of two polynomials $P_{L}(\omega)$ and $Q_{K}(\omega)$. Such ratios are given in terms of the signal points alone, $\left\{c_{n}\right\}(0 \leq n \leq N-1)$. The customary non-parametric processing (shape estimation) in the FPT is completed as soon as the complex envelope spectrum $P_{L}(\omega) / Q_{K}(\omega)$ is reconstructed. On the other hand, the parameter estimation by the FPT is performed by explicitly solving the problem of spectral analysis (the quantification problem). This is an inverse problem which, for the known set of signal points $\left\{c_{n}\right\}(0 \leq n \leq N-1)$, is tasked to reconstruct the $K$ fundamental frequencies $\left\{\omega_{k}\right\}$ and fundamental amplitudes $\left\{d_{k}\right\}$ that need to cohere with the 
quantum-mechanical prediction (2). In that process, the model order $K$ (the number of physical resonances) is also treated as yet another reconstruction parameter. Algorithmically, the parametric FPT first finds $\left\{\omega_{k}\right\}$ as the eigen-roots of the characteristic denominator polynomial $Q_{K}(\omega)$ by solving the so-called secular equation, $Q_{K}(\omega)=0$. Subsequently, the amplitudes $\left\{d_{k}\right\}$ are determined analytically as the Cauchy residues of e.g. the non-degenerate spectrum $P_{L}(\omega) / Q_{K}(\omega)$ with non-equal eigen-frequencies:

$$
\frac{P_{L}(\omega)}{Q_{K}(\omega)}=\sum_{k=1}^{K} \frac{d_{k}}{\omega-\omega_{k}}, d_{k}=\left[\frac{P_{L}(\omega)}{(\mathrm{d} / \mathrm{d} \omega) Q_{K}(\omega)}\right]_{\omega=\omega_{k}}
$$

Here, one eigen-amplitude $d_{k}$ corresponds to a single eigen-frequency $\omega_{k}$. In the degenerate version of the spectrum $P_{L}(\omega) / Q_{K}(\omega)$, some of the eigen-frequencies coincide. This causes more than one eigen-amplitude to correspond to a single eigenfrequency. If the multiplicity of the $k$ th eigen-frequencies $\omega_{k}$ is $m_{k}$, the Cauchy residue of $P_{L}(\omega) / Q_{K}(\omega)$ yields the degenerate eigen-amplitude $d_{k, m_{k}}$ as:

$$
d_{k, m_{k}}=\left[\frac{P_{L}(\omega)}{(\mathrm{d} / \mathrm{d} \omega)^{m_{k}} Q_{K}\left(\omega_{k}\right)}\right]_{\omega=\omega_{k}} .
$$

In this case, the Heaviside partial fraction decomposition of the degenerate spectrum $P_{L}(\omega) / Q_{K}(\omega)$ is:

$$
\frac{P_{L}(\omega)}{Q_{K}(\omega)}=\sum_{k=1}^{J} \sum_{m_{k}=1}^{\tilde{M}_{k}} \frac{d_{k, m_{k}}}{\left(\omega-\omega_{k}\right)^{m_{k}}}
$$

where $\tilde{M}_{k}=\max \left\{m_{k}\right\}, \tilde{M}_{1}+\tilde{M}_{2}+\ldots+\tilde{M}_{J}=K$, and $J$ is the total number of coincident eigen-frequencies $\omega_{k}$. This generalization of (7) means that the $k$ th eigenfrequency $\omega_{k}$ is repeated $m_{k}$ times $\left(m_{k}>1\right)$ in the spectrum $P_{L}(\omega) / Q_{K}(\omega)$ from (9). The lineshapes of non-degenerate and degenerate spectra are different in that the former (7) and the latter (9) are Lorentzian and non-Lorentzian functions, respectively.

\subsection{Explicit reformulation of signal processing in terms of quantum-mechanical spectral analysis}

The two pillars of the Schrödinger picture of quantum physics are the non-stationary (time-dependent) and stationary (time independent) Schrödinger equations, respectively:

$$
\begin{aligned}
& i \frac{\partial}{\partial t} \Phi(t)=\Omega \Phi(t), \\
& \Omega \Psi_{k}=\omega_{k} \Psi_{k}, 1 \leq k \leq K, \hbar=\frac{h}{2 \pi} \equiv 1,
\end{aligned}
$$


where $h$ is Planck's constant. In the so-called natural units, we have $\hbar=1$, in which case, frequency and energy are synonymous. Quantity $\Omega$ is the system's operator (dynamics generator), which in physics is a hermitean Hamiltonian $\left(\Omega^{\dagger}=\Omega\right.$ ) for stationary states with real eigen-energies or eigen-frequencies. Hamiltonian operators consist of the kinetic energy operators and the operators of the interaction potentials among the constituents of the system. For dissipative systems, $\Omega$ is non-hermitean $\left(\Omega^{\dagger} \neq \Omega\right)$ and $\omega_{k}$ is a complex eigen-value $\left(\omega_{k}=\operatorname{Re} \omega_{k}+i \operatorname{Im} \omega_{k}\right)$ of the eigenproblem (11). The eigen-function $\Psi_{k}$ describes the $k$ th state of the system. Stationary, stable states of infinite lifetime have line (or stick) spectra with zero-valued peak widths $\left(\operatorname{Im} \omega_{k}=0\right)$. Decaying or radiative states for non-hermitean $\Omega$ have spread-out peaks of non-zero widths $\left(\operatorname{Im} \omega_{k} \neq 0\right)$. These are metastable states that last for a certain finite period and then die out at the times longer than the reciprocal of their widths, $1 / \operatorname{Im} \omega_{k}$. The set of the eigen-solution $\left\{\omega_{k}, \Psi_{k}\right\}$ of the eigen-problem (11) is called a spectrum, in the terminology of mathematics. That is why obtaining the spectral set $\left\{\omega_{k}, \Psi_{k}\right\}$ is referred to as "solving the spectral analysis problem", or "spectral analysis", for short. Energies are quantized (discretized) in quantum physics. This means that transition from one to another stationary state is possible only if the external energy absorbed by the system is not smaller than the difference between the two considered energy levels. The main postulate of quantum physics is information completeness. It hypothesizes that everything one could possibly know (measure, compute) on a general system is contained in (11). Thus far, experimental measurements have abundantly and universally confirmed the validity of this hypothesis. This, in turn, justifies the foundation of quantum physics on the basis of the discrete transition energies (frequencies) and the underlying Schrödinger Eq. (11).

As to (10), the non-stationary state $\Phi(t)$ describes the time evolution of the system from a beginning instant (say $t=0$ ) with the initial configuration $\Phi_{0}$ (supposed to be known) to any subsequent time $t$. For conservative systems, $\Omega$ is time-independent, so that (10) can be solved, albeit formally, with the boundary condition $\Phi(0)=\Phi_{0}$ :

$$
\Phi(t)=\mathrm{U}(t) \Phi_{0}, \mathrm{U}(t)=e^{-i \Omega t},
$$

where $U(t)$ is the time evolution operator. Solution (12) of (10) is the cornerstone of the determinism of quantum physics. It tells us that for the known $\Omega$ and $\Phi_{0}$, the state of the system is completely determined by $\Phi(t)$. In other words, for the known initial state $\Phi_{0}$ at $t=0$, the state $\Phi(t)$ of the system at any subsequent time $t>0$ will also be known for the given $\Omega$. To this end, all that is needed is to prepare the initial state $\Phi_{0}$ of the system, as e.g. a well-localized wave-packet, and subsequently propagate it in time by means of (10) or, more explicitly, through the time evolution operator $U(t)$ from (12). Solution (12) is formal because the action of the exponential operator in $\mathrm{U}(t)$ must be defined and implemented.

Traditionally, physicists perform computations by solving either (10) or (11) separately. However, it is advantageous to combine (10) and (11) in the same computation. This is not only practical from the computational viewpoint, but it is also fundamental. It unifies the stationary and non-stationary representations into a single formalism which, in turn, exhibits all the facets of the two separate frameworks. This is accomplished by exploiting the mentioned completeness relation, which reads as: 


$$
\left.\sum_{k=1}^{K} \pi_{k}=\hat{1}, \pi_{k}=\mid \Psi_{k}\right)\left(\Psi_{k} \mid\right.
$$

where $\pi_{k}$ and $\hat{1}$ are the projection and unity operators, respectively. Equation (13) is also known as the closure formula. Here, the soft brackets are used to indicate that for non-hermitean operators, the scalar (or inner) products are symmetric, $(\chi \mid \xi)=(\xi \mid \chi)$. This differs from the Dirac nomenclature, $\langle\chi \mid \xi\rangle=\left\langle\xi^{*} \mid \chi^{*}\right\rangle$, where the star superscript symbolizes complex conjugation. Thus, using the identity $\mathrm{U}(t)=\hat{1} \cdot \mathrm{U}(t)$ in (12), it follows:

$$
\begin{aligned}
\mathrm{U}(t) & =\sum_{k=1}^{K} \pi_{k} \mathrm{e}^{-i \omega_{k} t}, \\
\Phi(t) & =\sum_{k=1}^{K} d_{k}^{1 / 2} \mathrm{e}^{-i \omega_{k} t} \Psi_{k}, \\
d_{k}^{1 / 2} & =\left(\Psi_{k} \mid \Phi_{0}\right) .
\end{aligned}
$$

It is seen that the single Eq. (15) incorporates both the non-stationary (10) and stationary (11) representations. It explicitly uses the completeness or closure relation (13) and, therefore, the entire information about the system is ingrained in (15). Moreover, the form (15) gives the explicit prescription for dealing with an exponential form of operator $\Omega$ in the evolution generator $\mathrm{U}(t)=\exp (-i \Omega t)$ from (12). The only physically-based prior information for (15) is the pair of the two main determinants of the system, $\left\{\Phi_{0}, \Omega\right\}$. This fundamental feature is carried over to any physical quantity derived from (15), such as the auto-correlation function defined by:

$$
c(t)=\left(\Phi(t) \mid \Phi_{0}\right) .
$$

The name auto-correlation function comes from the occurrence that the state $\Phi$ of the system correlates to itself at two different instances, $t$ and 0 . Upon insertion of $\Phi(t)$ from (15) into $c(t)$ from (17), it follows:

$$
\begin{aligned}
c(t) & =\sum_{k=1}^{K} d_{k} \mathrm{e}^{-i \omega_{k} t} ; \operatorname{Im}\left(\omega_{k}\right)<0, \\
d_{k} & =\left(\Psi_{k} \mid \Phi_{0}\right)^{2},
\end{aligned}
$$

where the symmetry of the scalar product is used, $\left(\Psi_{k} \mid \Phi_{0}\right)=\left(\Phi_{0} \mid \Psi_{k}\right)$. The result (18) is identical to (1). This establishes the equivalence between time signals and autocorrelation functions. The derivation of auto-correlation function (17) is performed using quantum physics. Therefore, the outlined formalism brings together quantum physics and the field of signal processing. Since quantum mechanics is a complete physics theory, such a merging enriches the status of signal processing to the level of quantum-mechanical signal processing [1]. 
Why would this matter? It matters immensely because signal processing traditionally introduces time signal (1) or (2) in an artificial manner, as an "ad hoc" mathematical model through a linear combination of complex damped harmonics. This empirical procedure is followed by imposing some arbitrarily chosen fitting constraints onto the set $\left\{\omega_{k}, d_{k}\right\}$ treated as the freely adjusting parameters. No such constraint needs to be associated with the input data for $c(t)$ when the time signal is from the onset based upon the quantum-mechanical auto-correlation function (18). This is the case because the measured input time signal itself already contains the entire information, which is fully captured by the completeness relation (13). The latter is wholly present, as per the outlined derivation, in the quantum-mechanical auto-correlation function (18). Overall, we see that rather than considering e.g. $\left\{d_{k}\right\}$ as an adjusting parameter in various fitting procedures, quantum mechanics gives the physical meaning to this quantity. As per the key relation $d_{k}=\left(\Psi_{k} \mid \Phi_{0}\right)^{2}$ from (19), the parameter $d_{k}$ represents the squared complex amplitude of the transition probability from the initial state $\Phi_{0}$ to the $k$ th eigen-state $\Psi_{k}$ of the system. In quantum-mechanical signal processing, the eigen-problem (11), or its equivalent $\mathrm{U}(\tau) \Psi_{k}=\mathrm{e}^{-i \omega_{k} \tau} \Psi_{k}$, where $\mathrm{U}(\tau)=\mathrm{e}^{-i \Omega \tau}$, is solved through diagonalization of $\Omega$ or $\mathrm{U}(\tau)$ on a given basis set of expansion functions $\left\{\zeta_{r}\right\}(r=1,2,3, \ldots)$. For example, $\zeta_{r}$ can be taken as $\Phi_{r}$, which is the discretized version of the Schrödinger state from (12), $\Phi_{r}=\mathrm{e}^{-i \Omega r \tau} \Phi_{0}$. In this latter basis, the general transition probability amplitude between any two states or configurations $\Phi_{r}$ and $\Phi_{s}$ is proportional to the matrix element $\left(\Phi_{r}|\mathrm{U}(\tau)| \Phi_{s}\right)=c_{r+s+1}$. Generally, even though the dynamic operators $\Omega$ and $U(\tau)$ are not given, the eigen-problems can, nevertheless, be solved if the matrix elements of these operators are known. In the Schrödinger basis $\left\{\Phi_{r}\right\}$, these matrix elements $\left(\Phi_{r}|\mathrm{U}(\tau)| \Phi_{s}\right)$ are simply the autocorrelation functions, or equivalently, the time signal points $c_{r+s+1}$, that are the known input data. This is precisely how quantum physics, in a "nutshell", intertwines with the field of signal processing. For in-depth exposition, see the textbook [1].

The frequency-domain counterpart of the time-domain auto-correlation function (18) is the Green function $G(\omega)$ given by the rational polynomial (6). Thus, the quantum-mechanical Green's function $G(\omega)$ is the paradiagonal Padé approximant, as the quotient of two polynomials $P_{K-1}(\omega) / Q_{K}(\omega)$.

The performed exposition explicitly shows several key characteristics:

(a) Quantum physics can be the backbone of signal processing,

(b) Quantum-mechanical cornerstone entities, the non-stationary and stationary Schrödinger equations, can also be the algorithms for spectral analysis in signal processing,

(c) Quantum-mechanical time-dependent auto-correlation functions are linear combinations of complex damped exponentials as the physically conceived time signals,

(d) Quantum-mechanical frequency spectra are the quotients of two polynomials that, by definition, are the Padé approximants, and

(e) Quantum-mechanical spectral analysis yielding $\left\{\omega_{k}, d_{k}\right\}$ necessitates both the eigen-frequencies $\omega_{k}$ and the eigen-functions $\Psi_{k}$ to be found. However, the PA avoids altogether reconstruction of $\Psi_{k}$ since the eigen-amplitudes are computed from (7) or (8) with no recourse to the eigen-functions, as opposed to (19). This is advantageous both computationally and for the reason of accuracy, because $\Psi_{k}$ 
are not variational, i.e. the eigen-functions contain first order errors, in contrast to the eigen-frequencies, $\omega_{k}$.

Thus, across interdisciplinary fields, any time signal taken to be a sum of complex attenuated harmonics, as in (2) will invariably yield the frequency spectrum in the explicit form of the Padé approximant via (4) or (6). This means that all the estimators using a time signal (2) to reconstruct the corresponding spectrum, in fact, try to compute the Padé approximants (4) or (6) with algorithms of varying complexity. However, the gist of the matter is in that for a time signal (2), the exact spectrum is the Padé approximant in the two equivalent representations, the Heaviside partial fractions (4) and its summed form - the polynomial quotient (6). So why not use the actual Padé approximants to arrive at the exact result with the most accurate/efficient/robust computational algorithm of linear algebra, consisting of solving a single system of linear equations for the expansion coefficients of the denominator polynomial, $Q_{K}(\omega)$ ? Let us take the FFT as an example to illustrate this point. Using time signal (2) as a set of the input numbers, the FFT evaluates the integral (3) approximately as a Riemann sum at a finite set of the Fourier grid frequencies. This gives the discrete Fourier transform (DFT) which is, in practice, computed by the Cooley-Tukey fast algorithm to yield the FFT. However, the Riemann sum itself is the least accurate numerical quadrature for the integral (3). On the other hand, the exact result for the same integral (3) is the PA from (4) or (6). Thus, by not accounting for the structure (2) of $c_{n}$, the FFT replaces the inherent Padé exact result (6) by its least accurate approximation- the Riemann sum. This is the origin of the low resolution of the Fourier analysis in signal processing.

\subsection{Derivative envelope spectra as differentiation transforms of conventional envelopes}

Spectrum $G(\omega)$ is a complex function of real independent variable $\omega$. For visualization purposes, its absorption or magnitude modes are usually plotted. By definition, the magnitude mode has no negative lobes, i.e. it is a spectrum with exclusively positivedefinite lineshapes for any phase $\varphi_{k}$ of $d_{k}$, where $d_{k}=\left|d_{k}\right| \exp \left(i \varphi_{k}\right)$. In general, and especially for encoded time signals, phases $\left\{\varphi_{k}\right\}$ of amplitudes $\left\{d_{k}\right\}$ are non-zero. This yields asymmetrical lineshapes with alternating polarity (peaks, dips), such that the real part $\operatorname{Re}(G(\omega))$ of spectrum $G(\omega)$ is invariably not positive-definite throughout the entire Nyquist range of frequencies. In such cases, the so-called zero- and/or firstorder phase corrections $\left(\mathrm{e}^{i \phi_{0}}, \mathrm{e}^{i \phi_{1}}\right)$ are usually introduced to multiply all the time signal points $\left\{c_{n}\right\}$ in an attempt to produce nearly positive-definite absorptive lineshapes. This cannot ever be fully satisfactory, since no two phase corrections can possibly make positive-definite the entire real part of a complex envelope. The reason is that each of the $K$ phases $\left\{\varphi_{k}\right\}(1 \leq k \leq K)$ of $d_{k}$ determines the overall lineshape of an envelope through their constructive and destructive interference.

Total shape spectra in either the absorption or magnitude modes are insufficiently informative for dense spectra with overlapping resonances, especially when these are strongly coupled together in their close proximity. One way to try to circumvent this obstacle is to go beyond envelopes and reconstruct the underlying components 
through parametric processing, i.e. quantification. An alternative way is to use only shape estimation to make the differential transform $G^{(m)}(\omega)$ of the non-parametrically computed envelope $G(\omega)$ :

$$
G^{(m)}(\omega)=\left(\frac{\mathrm{d}}{\mathrm{d} \omega}\right)^{m} G(\omega)
$$

where $m$ is a positive integer $(m=1,2,3, \ldots)$. The goal is to see whether for the seed envelope spectrum $G(\omega)$, generated by means of the non-parametric FPT, the resulting derivative spectra $G^{(m)}(\omega)$ could be capable of yielding accurate peak parameters (positions, widths, heights). This novel version of the FPT is termed the derivative fast Padé transform, dFPT.

Specifically, despite relying upon purely non-parametric processing for shape estimation (i.e. without ever resorting to the solution of the quantification problem), we want to find out whether the dFPT could be capable not only of visually disentangling tightly overlapping peaks, but also of exactly reconstructing their positions, widths and heights. This task will be carried out by applying the dFPT to time signals typical of those encoded by MRS for breast cancer. This modality of MRS and MRSI is called derivative magnetic resonance spectroscopy (dMRS) and derivative magnetic resonance spectroscopic imaging (dMRSI), respectively.

\subsubsection{Noise in the derivative Fourier spectral envelopes}

For the time signal $c(t)$ from (1) of a hypothetical infinite duration $(T=\infty)$, the $m$ th derivative $F^{(m)}(v)$ of the one-sided Fourier integral from the rhs of (3) reads as:

$$
F^{(m)}(v) \equiv\left(\frac{\mathrm{d}}{\mathrm{d} v}\right)^{m} F(v)=\int_{0}^{\infty} \mathrm{d} t \mathrm{e}^{2 \pi i v t}\left\{(2 \pi i t)^{m} c(t)\right\}
$$

From here, for any realistic time signal of finite duration $(T<\infty)$, upon truncation of the upper integration limit from $t_{\max }=\infty$ to $t_{\max }=T$, the derivative discrete Fourier transform (dDFT), amenable to signal processing, is given by:

$$
\begin{aligned}
F_{k}^{(m)} \equiv\left(\frac{\mathrm{d}}{\mathrm{d} \nu}\right)^{m} F_{k} & =\sum_{n=0}^{N-1} \mathrm{e}^{2 \pi i n k / N} c_{n, m} ; c_{n, m}=(2 \pi i n \tau)^{m} c_{n} \\
F_{k} & =\sum_{n=0}^{N-1} \mathrm{e}^{2 \pi i n k / N} c_{n} .
\end{aligned}
$$

Here, $F_{k}$ from (22) is the customary (the zeroth-order derivative , $m=0$ ) DFT. As mentioned, in the DFT and dDFT, the Fourier grid frequencies are used. They are given by $\omega_{k}^{\mathrm{F}}=2 \pi k / T$ or $\nu_{k}^{\mathrm{F}}=k / T(\tau=T / N)$, where the angular or cyclic $(\omega)$ and the linear $(\nu)$ frequencies are connected by the usual relation $\omega=2 \pi \nu$. Thus, in the Fourier analysis, any spectrum is a stick spectrum which exists only on the 
Fourier grid frequencies $\left\{\omega_{k}^{\mathrm{F}}\right\}(0 \leq k \leq N-1)$. By contrast, in the Padé analysis, every spectrum exists at any frequency $\omega$ or $v$. The $m$ th derivative dDFT is generated by first multiplying the input time signal $c_{n}$ by the power function $(2 \pi i n \tau)^{m}$ before taking the discrete Fourier transform. With augmentation of time $t$, however, the power function $t^{m}$ in (21) amplifies noise in the product $t^{m} c(t)$. In other words, the multiplier $t^{m}(m=1,2, \ldots)$ of $c(t)$ in (21) weights more heavily at longer compared to shorter instants within the complete time duration $T$ of $c(t)$. For measured time signals, mainly noise is counted/recorded/encoded at longer instants, and, hence, the weight function $t^{m}$ enhances noise with increasing order $m$ of differentiation. Thus, the increased order of differentiation $m$ acts as a noise amplifier in the dDFT. Of course, the same obstacle also exists in the dFFT which is obtained by applying the Cooley-Tukey fast algorithm to the dDFT $[1,6]$.

\subsubsection{Noise in the Padé derivative spectral envelopes}

The stated severe problem with the power function $t^{m}$ in the dFFT does not exist at all in the dFPT. The reason is that the dFPT applies the operator $(\mathrm{d} / \mathrm{d} \nu)^{m}$ directly to the

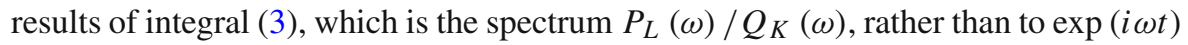
from the integrand of the same integral. This implies that the offending product $t^{m} c_{n}(t)$ is never set up in the dFPT. Instead, the unaltered input data set $\left\{c_{n}\right\}$ is used to generate the seed spectrum $P_{L}(\omega) / Q_{K}(\omega)$ to which the $m$ th derivative $(\mathrm{d} / \mathrm{d} \nu)^{m}$ is applied. In this way, the original noise from $\left\{c_{n}\right\}$ is treated by the non-derivative envelope $P_{L} / Q_{K}$ in the FPT, to which the differentiation transform is applied, yielding the dFPT. Thus, the additional noise through $t^{m} c_{n}(t)$ is never generated since the latter product is absent from the dFPT, as stated. It should be mentioned that both $P_{L} / Q_{K}$ and $(\mathrm{d} / \mathrm{d} \nu)^{m} P_{L} / Q_{K}$ are given by their analytical expression, such that no numerical differentiation (which is prone to errors) is ever used.

\section{Implementation of the theory}

Let us assume that we are given an exact Green's function, which is a total shape complex-valued spectrum (envelope):

$$
G\left(z^{-1}\right)=\sum_{n=0}^{\infty} c_{n} z^{-n}, c_{n}=\sum_{k=1}^{K} d_{k} \mathrm{e}^{i \omega_{k} n \tau}, \omega_{k}=2 \pi v_{k}, \operatorname{Im}\left(\omega_{k}\right)>0
$$

where $z^{-1}=\mathrm{e}^{-i \omega \tau}$. Note that in (24) we have a different convention for $\operatorname{Im}\left(\omega_{k}\right)$ relative to (1) or (2). In (24), the quantity $z^{-1}$ is the harmonic variable, and the discrete time signal points $\left\{c_{n}\right\}$ are the expansion coefficients in this MacLaurin power series of $G\left(z^{-1}\right)$. The diagonal fast Padé transforms, $\mathrm{FPT}^{( \pm)}$, are defined by the relationships: 


$$
\mathrm{FPT}^{( \pm)}: P_{K}^{ \pm}\left(z^{ \pm 1}\right) G\left(z^{-1}\right)-Q_{K}^{ \pm}\left(z^{ \pm 1}\right)=O^{ \pm}\left(z^{ \pm 2 K \pm 1}\right)
$$

where symbols $O^{ \pm}\left(z^{ \pm 2 K \pm 1}\right)$ are the remainders given by their series (to be identified later on), beginning with the terms $z^{ \pm 2 K \pm 1}$. In (25), quantities $P_{K}^{ \pm}\left(z^{ \pm 1}\right)$ and $Q_{K}^{ \pm}\left(z^{ \pm 1}\right)$ are polynomials of common degree $K$, defined as:

$$
P_{K}^{ \pm}\left(z^{ \pm 1}\right)=\sum_{r=r_{ \pm}}^{K} p_{r}^{ \pm} z^{ \pm r}, Q_{K}^{ \pm}\left(z^{ \pm 1}\right)=\sum_{s=0}^{K} q_{s}^{ \pm} z^{ \pm s}, r_{+}=1, r_{-}=0
$$

where $p_{r}^{ \pm}$and $q_{s}^{ \pm}$are the expansion coefficients. The mathematical symbols for the $\mathrm{FPT}^{( \pm)}$are $G_{K}^{ \pm}\left(z^{ \pm 1}\right)$ that are also called the Padé-Green functions. Neglect of $O^{ \pm}\left(z^{ \pm 2 K \pm 1}\right)$ in $(25)$ amounts to approximating therein the exact input function $G\left(z^{-1}\right)$ from (24) by $G_{K}^{ \pm}\left(z^{ \pm 1}\right)$ :

$$
\begin{aligned}
& P_{K}^{ \pm}\left(z^{ \pm 1}\right) G\left(z^{-1}\right)-Q_{K}^{ \pm}\left(z^{ \pm 1}\right) \approx 0, \\
& \therefore G\left(z^{-1}\right) \approx G_{K}^{ \pm}\left(z^{ \pm 1}\right), \\
& \therefore P_{K}^{ \pm}\left(z^{ \pm 1}\right) G_{K}^{ \pm}\left(z^{ \pm 1}\right)-Q_{K}^{ \pm}\left(z^{ \pm 1}\right)=0 .
\end{aligned}
$$

This procedure identifies the $\mathrm{FPT}^{( \pm)}$as the rational polynomials $G_{K}^{ \pm}\left(z^{ \pm 1}\right)$ deduced through division of both sides of (27c) by $Q_{K}^{ \pm}\left(z^{ \pm 1}\right) \neq 0$ :

$$
\mathrm{FPT}^{( \pm)}: G_{K}^{ \pm}\left(z^{ \pm 1}\right)=\frac{P_{K}^{ \pm}\left(z^{ \pm 1}\right)}{Q_{K}^{ \pm}\left(z^{ \pm 1}\right)}
$$

Note that working with the para-diagonal or diagonal forms of the $\mathrm{FPT}^{( \pm)}$is equivalent, as it is with any other polynomial degrees $L$ and $K$ in $P_{L}^{ \pm} / Q_{K}^{ \pm}$. The practical reasons for preferring to use $P_{K-1}^{ \pm} / Q_{K}^{ \pm}$or $P_{K}^{ \pm} / Q_{K}^{ \pm}$, i.e. $L=K-1$ (para-diagonal) or $L=K$ (diagonal) is that in these two cases the errors in the $\mathrm{FPT}^{( \pm)}$are the smallest and, moreover, convergence exhibits the best features [1].

In an analogy to the exact infinite-rank Green function $G\left(z^{-1}\right)$ from (24), we can also obtain the equivalent representations of the $\mathrm{FPT}^{( \pm)}$given by the power series expansions of $G_{K}^{ \pm}\left(z^{ \pm 1}\right)$ :

$$
G_{K}^{ \pm}\left(z^{ \pm 1}\right)=\sum_{m=0}^{\infty} a_{m}^{ \pm} z^{ \pm m}
$$

These are recognized as the MacLaurin series that are the developments of $G_{K}^{ \pm}\left(z^{ \pm 1}\right)$ around the points $z^{ \pm 1}=0$, respectively. The general analytical forms of the coefficients $a_{m}^{ \pm}$are defined by the $m$ th derivatives $\left(\mathrm{d} / \mathrm{d} z^{ \pm 1}\right)^{m}$ of $G_{K}^{ \pm}\left(z^{ \pm 1}\right)$ taken at the expansion points $z^{ \pm 1}=0$ of the series in (29): 


$$
a_{m}^{ \pm}=\frac{1}{m !}\left\{\left(\frac{\mathrm{d}}{\mathrm{d} z^{ \pm 1}}\right)^{m} G_{K}^{ \pm}\left(z^{ \pm 1}\right)\right\}_{z^{ \pm 1}=0}
$$

Using (27b) and comparing (24) with (29) gives, by reference to the theorem on the uniqueness of the power series expansion [1], the following relation:

$$
a_{m}^{ \pm}=c_{m}(0 \leq m \leq 2 K) \text {. }
$$

This means that the fast Padé transforms $\mathrm{FPT}^{( \pm)}$of order $K$ reconstruct exactly the first $2 K+1$ time signal points $\left\{c_{m}\right\}(0 \leq m \leq 2 K)$ from the single input series $G\left(z^{-1}\right)$ from (24). Moreover, this comparison permits identification of the remainders $O^{ \pm}$in (25) as:

$$
O^{ \pm}\left(z^{ \pm 2 K \pm 1}\right)=\sum_{m=2 K+1}^{\infty} a_{m}^{ \pm} z^{ \pm m}
$$

It is clear from here that indeed $O^{ \pm}\left(z^{ \pm 2 K \pm 1}\right)$ are the power series beginning with the terms $z^{ \pm 2 K \pm 1}$, as stated earlier.

Overall, it follows that the fast Padé transforms $\mathrm{FPT}^{( \pm)}$possess the following three important features:

(i) The expansion coefficients $\left\{a_{m}^{ \pm}\right\}$of $G_{K}^{ \pm}\left(z^{ \pm 1}\right)$ contain the identical information provided by the expansion coefficients $\left\{p_{r}^{ \pm}, q_{r}^{ \pm}\right\}$in the Padé rational polynomials $P_{K}^{ \pm}\left(z^{ \pm 1}\right) / Q_{K}^{ \pm}\left(z^{ \pm 1}\right)$ from (26).

(ii) The errors of the $\mathrm{FPT}^{( \pm)}$invoked by the passage from (25) to (27a) are the remainders $O^{ \pm}\left(z^{ \pm 2 K \pm 1}\right)$ that are obtainable from the analytical formulae in (32), once the expansion coefficients $\left\{a_{m}^{ \pm}\right\}$become available.

(iii) For the same truncation level of the input MacLaurin series in (24) and the two output Padé-MacLaurin series in (29), the developments from (29) contain twice more information, which testifies to the extrapolation capability (i.e. the predictive power) of the $\mathrm{FPT}^{( \pm)}$.

All three features (i)-(iii) of the $\mathrm{FPT}^{( \pm)}$are rooted in the expansion coefficients $\left\{a_{m}^{ \pm}\right\}$ of $G_{K}^{ \pm}\left(z^{ \pm 1}\right)$ from (29). Therefore, it would be important to investigate the nature of these coefficients to see whether they could possibly yield further information beyond (i)-(iii). This goal is motivated by the fact that the sets $\left\{a_{m}^{ \pm}\right\}$are, in fact, never explicitly generated in applications of the $\mathrm{FPT}^{( \pm)}$. By the definitions in (30), it follows that $a_{m}^{ \pm}$for any fixed $m$ can give the local information, i.e. the information at the expansion points $z^{ \pm 1}=0$ alone. However, the sets $\left\{a_{m}^{ \pm}\right\}(m=0,1,2, \ldots)$, by way of series in (29) are traced back to the Padé quotients $P_{K}^{ \pm}\left(z^{ \pm 1}\right) / Q_{K}^{ \pm}\left(z^{ \pm 1}\right)$ that themselves yield the global information, i.e. the information in the entire complex $z^{ \pm 1}$ planes, respectively. Both representations $P_{K}^{ \pm}\left(z^{ \pm 1}\right) / Q_{K}^{ \pm}\left(z^{ \pm 1}\right)$ in (28) and the Padé-MacLaurin series in (29) are analytical functions of $z^{ \pm 1}$, meaning that they are continuous and differentiable any number of times with respect to variables $z^{ \pm 1}$. In fact, it is this analyticity, with the accompanying differentiability, that provides the general expansion coefficients $a_{m}^{ \pm}$as 
the $m$ th derivatives of $P_{K}^{ \pm}\left(z^{ \pm 1}\right) / Q_{K}^{ \pm}\left(z^{ \pm 1}\right)$ taken at $z^{ \pm 1}=0$. Thus, every coefficient $a_{m}^{+}$or $a_{m}^{-}$, being itself a provider of local information, when collected in the assemblies $\left\{a_{m}^{+}\right\}(m=0,1,2, \ldots)$, or $\left\{a_{m}^{-}\right\}(m=0,1,2, \ldots)$, can yield the global information about the entire spectra (response functions) by way of $G_{K}^{ \pm}\left(z^{ \pm 1}\right)$. In other words, it is entirely equivalent to give either $P_{K}^{ \pm}\left(z^{ \pm 1}\right) / Q_{K}^{ \pm}\left(z^{ \pm 1}\right)$ or, alternatively, the expansion coefficients $\left\{a_{m}^{ \pm}\right\}$to be used in the series in (29). This equivalence is simply an extension of the well-known fact that an ordinary classical polynomial (Legendre, Laguerre, Hermite, Gegenbauer,...), say $V_{M}(u)$, can equivalently be introduced either through its generating closed formula or via the recursion relation or by a set of the known expansion coefficients $\left\{v_{m}\right\}$ to be used in the power series $V_{M}(u)=\sum_{m=0}^{M} v_{m} u^{m}$. Note also that according to the generalized Weierstrass theorem [7], every function can be expanded to within arbitrary accuracy in terms of rational polynomials, i.e. the Padé approximants. This is an extension of the usual Weierstrass theorem according to which a linear combination of ordinary polynomials can be used for precise representations of a given function. The advantage of the generalized Weierstrass theorem is that the basis of rational polynomials applies to a wider class of functions that possess singularities, such as poles, branch points and branch cuts [1].

The key question which arises from this parallel is: would it then be possible to glean global information by evaluating the inner part $\left(\mathrm{d} / \mathrm{d} z^{ \pm 1}\right)^{m} G_{K}^{ \pm}\left(z^{ \pm 1}\right)$ of the curly brackets in (30) at any $z^{ \pm 1}$, instead of merely at $z^{ \pm 1}=0$ (the latter points are used to generate $\left.a_{m}^{ \pm}\right)$? The answer to this question is in the affirmative. To explore this novel avenue, we shall analyze the $m$ th derivative spectra in the $\mathrm{FPT}^{( \pm)}$defined at any $z^{ \pm 1}$ by:

$$
G_{K}^{ \pm(m)}\left(z^{ \pm 1}\right)=\left(\frac{\mathrm{d}}{\mathrm{d} v}\right)^{m} G_{K}^{ \pm}\left(z^{ \pm 1}\right)
$$

This introduces the derivative fast Padé transforms, $\mathrm{dFPT}^{( \pm)}$, in the following explicit forms:

$$
\mathrm{dFPT}^{( \pm)}: \quad G_{K}^{ \pm(m)}\left(z^{ \pm 1}\right)=\left(\frac{\mathrm{d}}{\mathrm{d} \nu}\right)^{m} \frac{P_{K}^{ \pm}\left(z^{ \pm 1}\right)}{Q_{K}^{ \pm}\left(z^{ \pm 1}\right)}
$$

Operationally, the $\mathrm{dFPT}^{( \pm)}$are accomplished in two steps. The first step consists of the standard $\mathrm{FPT}^{( \pm)}$that use the Green's polynomial $G_{N}\left(z^{-1}\right)$ defined by:

$$
G_{N}\left(z^{-1}\right) \equiv \sum_{n=0}^{N-1} c_{n} z^{-n}
$$

where $c_{n}$ is taken from (24). This is a truncated form of the Green's function $G\left(z^{-1}\right)$ from (24) to be used to extract the expansion coefficients $\left\{p_{r}^{ \pm}, q_{s}^{ \pm}\right\}$of the polynomials $\left\{P_{K}^{ \pm}, Q_{K}^{ \pm}\right\}$, respectively. The second step in the $\mathrm{dFPT}^{( \pm)}$is to compute the $m$ th derivative spectra $G_{K}^{ \pm(m)}\left(z^{ \pm 1}\right)$ using (33) for the sets $1 \leq m \leq M^{ \pm}$, where $M^{ \pm}$are any 
positive integers. These computations, in both the 1 st and the 2 nd step , can be performed either for a single model order $K$ or for a set of values $K=K_{\min }(\Delta K) K_{\max }$, i.e. $K=K_{\min }, K_{\min }+\Delta K, K_{\min }+2 \Delta K, \ldots, K_{\max }$, where $\Delta K$ is a fixed rate of successively increasing $K_{\min }$. Recall that in the 1st step, employing the standard $\mathrm{FPT}^{( \pm)}$, both sets of Padé polynomials $P_{K}^{ \pm}\left(z^{ \pm 1}\right)$ and $Q_{K}^{ \pm}\left(z^{ \pm 1}\right)$ are extracted from the definition of the type (27a) where $G\left(z^{-1}\right)$ from (24) is replaced by its truncated version $G_{N}\left(z^{ \pm 1}\right)$ from (35) via:

$$
P_{K}^{ \pm}\left(z^{ \pm 1}\right) G_{N}\left(z^{-1}\right)=Q_{K}^{ \pm}\left(z^{ \pm 1}\right)
$$

To this end, using (36), only a single system of linear equations $\sum_{s=0}^{K} q_{s}^{+} c_{m+s}=0$ or $\sum_{s=0}^{K} q_{s}^{-} c_{m+k-s}=0$ is solved in the $\mathrm{FPT}^{(+)}$or $\mathrm{FPT}^{(-)}$, respectively, to generate the expansion coefficients $\left\{q_{s}^{ \pm}\right\}(0 \leq s \leq K)$ of the denominator polynomials $Q_{K}^{ \pm}\left(z^{ \pm 1}\right)$. The coefficients $\left\{p_{r}^{ \pm}\right\}$of the numerator polynomials $P_{K}^{ \pm}\left(z^{ \pm 1}\right)$ are obtained from their analytical expressions in terms of $\left\{c_{n}, q_{s}^{ \pm}\right\}$as $p_{m}^{+}=\sum_{r=0}^{K-m} c_{r} q_{r+m}^{+}$and $p_{m}^{-}=$ $\sum_{r=0}^{m} c_{r} q_{m-r}^{-}$, respectively [8].

Once the spectra $P_{K}^{ \pm}\left(z^{ \pm 1}\right) / Q_{K}^{ \pm}\left(z^{ \pm 1}\right)$ in the $\mathrm{FPT}^{( \pm)}$become available in the explained way, the response functions $\mathrm{dFPT}^{( \pm)}$are completed by generating the $m$ th derivatives of $P_{K}^{ \pm}\left(z^{ \pm 1}\right) / Q_{K}^{ \pm}\left(z^{ \pm 1}\right)$ with respect to $v$. The ensuing $m$ th derivative spectra $G_{K}^{ \pm(m)}\left(z^{ \pm 1}\right)$ from (34) in the $\mathrm{dFPT}^{( \pm)}$are computed from the general analytical formulae for $(\mathrm{d} / \mathrm{d} v)^{m} P_{L}^{ \pm}\left(z^{ \pm 1}\right) / Q_{K}^{ \pm}\left(z^{ \pm 1}\right)$ for an arbitrary positive integer $m$ and for any degrees $L$ and $K$ of polynomials $P_{L}^{ \pm}\left(z^{ \pm 1}\right)$ and $Q_{K}^{ \pm}\left(z^{ \pm 1}\right)$. This analytical formula is expressed through the Bell polynomials [9]. As stated, the diagonal forms $\mathrm{dFPT}^{( \pm)}$from (34) are specified for the same degree of the numerator and denominator polynomials, i.e. for $L=K$. This sets the stage for implementation of the $\mathrm{dFPT}^{( \pm)}$, the outcomes of which are found in the Results section.

Importantly, throughout this section, the focus is on the derivatives $G_{K}^{ \pm(m)}\left(z^{ \pm 1}\right)$ of the total shape spectra or envelopes $P_{K}^{ \pm}\left(z^{ \pm 1}\right) / Q_{K}^{ \pm}\left(z^{ \pm 1}\right)$. The seed spectra $P_{K}^{ \pm}\left(z^{ \pm 1}\right) / Q_{K}^{ \pm}\left(z^{ \pm 1}\right)$ are supposed to be generated by the non-parametric $\mathrm{FPT}^{( \pm)}$. Therefore, the ensuing derivative spectra $(\mathrm{d} / \mathrm{d} v)^{m} P_{K}^{ \pm}\left(z^{ \pm 1}\right) / Q_{K}^{ \pm}\left(z^{ \pm 1}\right)$ in the $\mathrm{dFPT}^{( \pm)}$remain within the same realm of non-parametric estimations. In general, as mentioned, non-parametric estimators are qualitative, since they are restricted to predictions of sole lineshapes of resonances. The main interest in introducing the $\mathrm{dFPT}^{( \pm)}$for $m>0$ is to see whether a "quantum leap" would be possible by obtaining the exact quantitative information (peak positions, widths, heights) for envelopes with overlapping peaks through exclusively shape estimations. In the present illustrations of the $\mathrm{dFPT}^{( \pm)}$the main focus is placed upon MRS. However, since time signals of the type from (24) are ubiquitous across interdisciplinary fields, the $\mathrm{dFPT}^{( \pm)}$can also be applied to the other kind of earlier mentioned spectroscopies. Likewise, this can be done in a number of different investigative areas that search the inner structure of substances e.g. phenomena on powder diffraction [10], X-ray photo-electron spectroscopy [11], etc. 


\section{Results}

\subsection{The input data}

As stated earlier, the present illustrations are concerned with synthesized (or simulated) complex-valued time signals, or equivalently, free induction decay (FID) curves. Both noise-free and noise-corrupted FIDs are considered herein, in order to systematically benchmark the derivative fast Padé transform, dFPT.

\subsubsection{Noiseless time signals}

A noiseless FID, $\left\{c_{n}\right\}$, of length $N$ is of the form given in (24). Therein, the fundamental parameters, the complex eigen-frequencies $\left\{v_{k}\right\}$ and complex amplitudes $\left\{d_{k}\right\}$ are selected to correspond to the specific in vitro MRS time signals encoded from breast cancer tissue obtained from patients undergoing surgical treatment, as reported in Ref. [12]. They read as:

$$
\begin{aligned}
\mathrm{M}_{k} & =\{\mathrm{Lac}, \text { Ala, Cho, PC, PE, GPC, } \beta-\text { Glc, Tau, } \mathrm{m}-\mathrm{Ins}\}, \\
\operatorname{Re}\left(v_{k}\right) & =\{1.332,1.471,3.212,3.220,3.221,3.232,3.251,3.273,3.281\} \mathrm{ppm}, \\
\operatorname{Im}\left(v_{k}\right) & =0.0008 \mathrm{ppm}(1 \leq k \leq K) ; \operatorname{Im}\left(v_{k}\right)>0, \\
\left|d_{k}\right| & =\{0.325,0.032,0.004,0.012,0.090,0.009,0.029,0.012,0.036\} \mathrm{au}, \\
\varphi_{k} & =0 \operatorname{rad}(1 \leq k \leq K),
\end{aligned}
$$

where $\mathrm{M}_{k}$ is the $k$ th metabolite, $K=9, d_{k}=\left|d_{k}\right| \mathrm{e}^{i \varphi_{k}}, \varphi_{k}$ is the amplitude phase, presently taken as $\varphi_{k}=0 \mathrm{rad}(1 \leq k \leq 9)$, and au denote arbitrary units.

\subsubsection{Specifics of the encoded time signals on which the simulation is based}

The FIDs from Ref. [12] were encoded at a high static magnetic field, about 14 tesla $(\mathrm{T})$, i.e. $B_{0}=14.1 \mathrm{~T}$ which corresponds to the precession Larmor frequency $\left(v_{\mathrm{L}}\right)$ of $600 \mathrm{MHz}$. The bandwidth (BW) was $6 \mathrm{kHz}$, which for a complex FID gives the sampling time $\tau=1 / \mathrm{BW} \approx 0.1667 \mathrm{~ms}$. The total signal length $N^{\prime}$ was $N^{\prime}=2^{16}=$ 65536, which yields the total acquisition time $T^{\prime}$ as $T^{\prime}=10.923 \mathrm{~s}$. In Ref. [12], the chemical shifts and concentrations of 9 metabolites were assigned to the metabolites listed in the 1 st row of (37). Metabolite concentrations $\mathrm{C}_{k}$ were expressed in terms of a reference concentration $\mathrm{C}_{\text {ref }}$. For the reference substance, resonating at zero frequency, $v=0$, a molecule (not present in the tissue) was taken to be 3-(trimethylsilyl-) 3,3,2,2-tetradeutero-propionic acid (TSP), with $\mathrm{C}_{\text {ref }}=0.05 \mathrm{mM} / \mathrm{g}$ (millimole per gram) of the wet weight (ww) of the scanned tissue. As such, the magnitude $\left|d_{k}\right|$ and $\mathrm{C}_{k}$ are connected with the relation $\left|d_{k}\right|=\mathrm{C}_{k} /(25 \mu \mathrm{M} / \mathrm{g})$, where $\mu \mathrm{M}$ is micromole. Conversely, for the given $\left|d_{k}\right|$, the metabolite concentration are obtained from the expression:

$$
\mathrm{C}_{k}=\mathrm{A}_{k} \mathrm{C}_{\mathrm{ref}}
$$


where $\mathrm{A}_{k}$ is the area of the $k$ th absorptive Lorentzian. The area, a Lorentzian lineshape, is given by:

$$
\mathrm{A}_{k}=\frac{\left|d_{k}\right|}{2}
$$

The $k$ th peak height $h_{k}$ is the ratio of $\left|d_{k}\right|$ and the full width at half maximum $\{\mathrm{FWHM}\}_{k}$ (proportional to $\operatorname{Im} v_{k}$ ) of the same Lorentzian:

$$
h_{k}=\frac{\left|d_{k}\right|}{\xi_{k}}, \xi_{k} \equiv\{\mathrm{FWHM}\}_{k} .
$$

For the input spectral parameters from (37), the peak heights, full widths at half maximae, areas and concentrations are listed as follows:

$$
\begin{aligned}
\mathrm{M}_{k}= & \{\mathrm{Lac}, \text { Ala, Cho, PC, PE, GPC, } \beta \text {-Glc, Tau, m-Ins }\}, \\
\operatorname{Re}\left(v_{k}\right)= & \{1.332,1.471,3.212,3.220,3.221,3.232,3.251,3.273,3.281\} \mathrm{ppm}, \\
h_{k}= & \{691.150,62.832,7.854,23.562,176.715,17.671,56.941, \\
& 219.911,70.686\} \mathrm{au}, \\
\{\mathrm{FWHM}\}_{k}= & 0.001257 \mathrm{ppm}(1 \leq k \leq 9), \\
\mathrm{C}_{k}= & \{8.125,0.8,0.1,0.3,2.25,0.285,0.725,2.8,0.9\} \mu \mathrm{M} / \mathrm{g},
\end{aligned}
$$

where for an easier mapping, the top 2 rows of (37) are repeated.

\subsubsection{Theoretical noise model for the encoded time signals}

All encoded FIDs are noise-contaminated. Noise can stem from many sources. Taken as a collective noise, such perturbations of physical time signals can, to a large extent, be viewed as random noise. Therefore, it is appropriate to model the encoded FIDs as the noiseless complex time signals $\left\{c_{n}\right\}(0 \leq n \leq N-1)$ additively modified as $c_{n}+r_{n}$. Here, the random numbers $\left\{r_{n}\right\}(0 \leq n \leq N-1)$ are taken as the complex Gaussian zero-mean white noise (orthogonal in the real and imaginary parts) of fixed prescribed standard deviations. We express a chosen standard deviation $\sigma$ as the product of the noise level $\lambda$ and the root-mean-square (RMS) error of the noiseless FID, $\left\{c_{n}\right\}$. The RMS is the quadratic mean of the noiseless magnitude $\left|c_{n}\right|$ of $c_{n}$, as given by:

$$
\begin{aligned}
\sigma & =\lambda \cdot \mathrm{RMS}_{0} \\
\mathrm{RMS}_{0} & =\left(\frac{1}{N} \sum_{n=0}^{N-1}\left|c_{n}\right|^{2}\right)^{1 / 2}
\end{aligned}
$$

where the subscript " 0 " in (42) and (43) indicates that this RMS is for the noiseless FID, $\left\{c_{n}\right\}$, with $\sigma=0$. In the present noise model, the noise level $\lambda$ in a noisy time signal $\left\{c_{n}+r_{n}\right\}$ is expressed by percentage of the $\mathrm{RMS}_{0}$ of the noiseless FID, $\left\{c_{n}\right\}$, via (42) and (43). Thus, stating that a noisy FID, $\left\{c_{n}+r_{n}\right\}$, has e.g. $10 \%$ noise contamination 
signifies that the noisy $\mathrm{RMS}_{\sigma}(\sigma \neq 0)$ is $10 \%$ of "the noiseless root-mean-square", $\mathrm{RMS}_{0}$, or equivalently:

$$
\mathrm{RMS}_{\sigma}=0.1 \mathrm{RMS}_{0}
$$

Note that it is appropriate to measure the noise level using the RMS error because it reduces the bias of the estimated noise variance. Moreover, this noise quantifier converts the signal oscillations to the variations of the power (or total energy) of the signal across the entire bandwidth, BW. In such a way, the RMS is seen to be a measure of the dynamics of the time signal.

\subsubsection{Total length of the synthesized time signals}

Given the abundantly established high-resolution capabilities of the non-parametric and parametric FPT [1,5,13-22], it is not necessary to presently perform exhaustive sampling of the input time signals. This applies to both synthesized and encoded FIDs. Thus, we are justified in severely truncating the long FID length $N^{\prime}=65536$ from encoding in Ref. [12], where the total acquisition time $T^{\prime}$ was $T^{\prime}=N^{\prime} \tau=10.923 \mathrm{~s}$, as stated. Thus, we sample the set $\left\{c_{n}\right\}$ with $c_{n}$ from (24) by using a total signal length $N$ which is 32 times shorter than $N^{\prime}$, i.e. $N=N^{\prime} / 32$, so that $N=2048$. By keeping the same BW as in Ref. [12], $\tau$ (in s) $=1 / 6000$, the total duration $T$ of the simulated time signal becomes a fraction of a second (s), relative to about eleven seconds for $T^{\prime}$ [12], i.e., $T=N \tau=2048 /(6000 \mathrm{~Hz}) \approx 0.341 \mathrm{~s}$.

\subsubsection{Dimensionless frequencies}

Throughout MRS and indeed nuclear magnetic resonance (NMR) spectroscopy, frequency is given in dimensionless units, parts per million, ppm. Resonance frequencies are proportional to the static magnetic field strength $B_{0}$. Thus, expressing resonances in hertz $(\mathrm{Hz})$ would render cumbersome and impractical comparisons of spectra computed with FIDs encoded at different values of $B_{0}$. On the other hand, when ppm are used to measure frequencies, the locations of the peaks of all the metabolites are always the same regardless of $B_{0}$. The reason for which such a miniscule order of magnitude $\left(10^{-6}\right)$ is chosen to measure frequencies through ppm is in the very nature of MRS and NMR spectroscopy. Larmor precession frequencies of the bulk magnetization vector $\vec{M}$ of the scanned sample around $\vec{B}_{0}$ are of the order of $10^{6} \mathrm{~Hz}$ (e.g. $v_{\mathrm{L}}=63.864 \mathrm{MHz}$ for clinical MR scanners with $B_{0}=1.5 \mathrm{~T}$ ). On the other hand, e.g. resonance widths are usually of the order of $\mathrm{Hz}$, i.e. a millionth fraction of Larmor frequencies. The weakness of the overall physical effect from e.g. proton MRS and the ensuing encoded time signal or FID comes from the occurrence that MR relies upon a tiny difference ( 1 spin in $10^{6}$ ) of the spin population of the only two quantum-mechanically allowed orientations of $\vec{M}$ (parallel and anti-parallel to $\vec{B}_{0}$ ).

Any real sweep frequency $v$ given in $\mathrm{Hz}$ is transferred to the dimensionless frequency unit in ppm by the relation: 


$$
v(\mathrm{ppm})=v_{\mathrm{H}_{2} \mathrm{O}}(\mathrm{ppm})-\frac{v}{v_{\mathrm{L}}}
$$

where $v_{\mathrm{L}}$ is the Larmor frequency in $\mathrm{MHz}$, and $\nu_{\mathrm{H}_{2} \mathrm{O}}(\mathrm{ppm})$ is the resonance frequency of water, $\mathrm{H}_{2} \mathrm{O}$. We set $\nu_{\mathrm{H}_{2} \mathrm{O}}=4.68 \mathrm{ppm}$ in (45), as this is the customary value. Note that $\nu_{\mathrm{H}_{2} \mathrm{O}}(\mathrm{ppm})$ may be different for changing temperature of the surrounding environment ( $v_{\mathrm{H}_{2} \mathrm{O}}=4.68 \mathrm{ppm}$ usually corresponds to room temperature). Both the sweep frequency $v$ and the real fundamental or eigen-frequencies (resonant or nodal, natural frequencies) that are chemical shifts, $\operatorname{Re}\left(v_{k}\right)$, are expressed in ppm through (45). On the other hand, the fundamental imaginary frequencies, $\operatorname{Im}\left(v_{k}\right)$, given in $\mathrm{Hz}$ are converted to ppm without any translational scaling factors:

$$
\operatorname{Im}\left(v_{k}\right)(\mathrm{ppm})=\frac{\operatorname{Im}\left(v_{k}\right)(\mathrm{Hz})}{v_{\mathrm{L}}} .
$$

\subsection{Output data (reconstructed spectra)}

As per the input parameters listed in (37), there are two distinct and well-separated frequency bands assigned to different metabolite molecules. On the far right band, there exist lactate (Lac) and alanine (Ala), at 1.332 and $1.471 \mathrm{ppm}$, respectively. The Lac resonance is the most abundant in the entire zoomed frequency window $v \in[1.332,3.281] \mathrm{ppm}$, and its concentration is $8.125 \mu \mathrm{M} / \mathrm{g}$ of ww of tissue, as seen in (41). In the 2nd band, which is a narrowly congested frequency interval $v \in[3.212,3.281] \mathrm{ppm}$, there are 7 metabolites: choline (Cho, $3.212 \mathrm{ppm}$ ); phosphocholine, $\mathrm{PC}$ (3.220 ppm); phosphoethanolamine, PE (3.221 ppm); glycerophosphocholine (GPC, $3.232 \mathrm{ppm})$; beta-glucose ( $\beta$-Glc, $3.251 \mathrm{ppm})$; taurine (Tau, $3.273 \mathrm{ppm}$ ) and myo-Inositol (m-Ins, $3.281 \mathrm{ppm})$. In this band, the most abundant metabolite is Tau with concentration $2.8 \mu \mathrm{M} / \mathrm{g}$ of $\mathrm{ww}$ of tissue, as per (41). The smallest chemical shift difference between any two adjacent metabolites in (37) or (41) is $0.001 \mathrm{ppm}$, and this occurs between PC and PE. These latter two metabolites sharply differ in their abundance. Their respective concentrations are: $\mathrm{C}_{\mathrm{PC}}=$ $0.3 \mu \mathrm{M} / \mathrm{g}$ and $\mathrm{C}_{\mathrm{PE}}=2.25 \mu \mathrm{M} / \mathrm{g}$ of ww of tissue. Since all the resonances have the same widths, while the magnitude $\left|d_{\mathrm{PE}}\right|=0.090$ au is larger than $\left|d_{\mathrm{PC}}\right|=0.012 \mathrm{au}$, it follows that the PE peak is by a factor of 7.5 taller $\left(h_{\mathrm{PE}}=176.715 \mathrm{au}\right)$ than that of $\mathrm{PC}\left(h_{\mathrm{PC}}=23.562 \mathrm{au}\right)$. These introductory remarks should facilitate inspection and quantitative visual comparisons of the PC and PE peak heights after their splitting in the Padé-generated derivative total shape spectra. In the dFPT, our main focus is precisely on the tightly overlapped PC and PE resonances. This is the case because PC is wholly swamped by its dominant neighbor PE, and our principal task in the present work is to see whether the derivative spectral envelopes in the dFPT can split PC and $\mathrm{PE}$ apart, and correctly quantify them, by determining their locations on the chemical shift axis, as well as their associated widths and peak heights.

We shall sub-divide the upcoming analysis of the reconstruction findings from the output data into two different Sects. 4.2.1 and 4.2.2. The first part (Sect. 4.2.1) deals with the noiseless and the other (Sect. 4.2.2) with the noisy input time signals from which the spectral envelopes are computed. The FPT and dFPT are used for both 
noise-free and noise-corrupted FIDs, whereas the dFFT is employed only for the most critical noisy part of the reconstructions. The noiseless reconstructions are important in benchmarking the derivative fast Padé transform, dFPT, for the exact input data with no perturbations. Such a procedure establishes the standard and unequivocal validation of this novel variant of the FPT. This is critical, because proceeding straight to the simulated noisy time signals or encoded FIDs would lead to ambiguities. In such cases, one would not know with certainty whether any encountered inaccuracy stems from the perturbed input FIDs or from some potential inadequacies of the applied signal processor. Only after successfully passing the rigorous scrutiny of the fully-controlled noiseless input FIDs, can an estimator be viewed as qualified to proceed further with its applications to synthesized noisy FIDs and then, finally, to encoded time signals.

Reconstructions with simulated noise-contaminated input FIDs are key for the validity of any signal processor. Here, the input data are still under control, since the entry spectral parameters to be reconstructed are given and the added noise level is also known. The challenge is to see how the same estimator performs under the influence of random perturbations of the input FIDs. Would it still be possible with the noise simulated FIDs, as well, to correctly retrieve all the input parameters. These are questions of practical importance, because any measurement, including encoded FIDs from MRS contains noise with varying extent. As stated earlier, the presently simulated input time signals are generated in relation to the FIDs from Ref. [12], encoded in vitro with a strong magnetic field, $B_{0}=14.1 \mathrm{~T}$. In this case, SNR is high, i.e. noise is mild. This fact permits corruption of the simulated noiseless input time signals $\left\{c_{n}\right\}$ with random Gaussian white noise of relatively lower standard deviation $\sigma$. Still, $\sigma$ must be reasonably sufficient to allow the effect of noise to take place, in comparisons between the dFPT and dFFT. This is particularly important for the dFPT, in order to see whether it can overcome the already mentioned basic defect of the dFFT, which amplifies noise relative to its conventional non-derivative counterpart, FFT.

In this Results section, the FPT and dFPT will be presented in their variants $\mathrm{FPT}^{(-)}$ and $\mathrm{dFPT}^{(-)}$, respectively. The same conclusions have also been drawn using the $\mathrm{FPT}^{(+)}$and $\mathrm{dFPT}^{(+)}$. The number of illustrations will thus not be doubled.

In Sect. 4.2.1, as mentioned, we will use the synthesized noiseless FID, $\left\{c_{n}\right\}$. This FID will be sampled employing $N=2048$ and $\tau$ (in s) $=1 / 6000$ with $v_{\mathrm{L}}=600 \mathrm{MHz}$, to cohere with Ref. [12]. Sampling of each time signal point is done by using $c_{n}$ from (24) with the fundamental parameters $\left\{v_{k}, d_{k}\right\}(1 \leq k \leq 9)$ from (37) with the convention $\operatorname{Im}\left(v_{k}\right)>0$. In Ref. [12], resonance widths were not reported. In (37), we have set all the linear imaginary fundamental frequencies to a common, fixed value, $\operatorname{Im}\left(v_{k}\right)=0.0008 \mathrm{ppm}(1 \leq k \leq 9)$. Thus, all the resonances have the same full width at half maximum, $\{\mathrm{FWHM}\}_{k}=0.001257 \mathrm{ppm}(1 \leq k \leq 9)$, as in (41).

In Sect. 4.2.2, the noiseless set $\left\{c_{n}\right\}$ will be perturbed by the mentioned random Gaussian white noise $\left\{r_{n}\right\}$, to yield the noisy time signal $\left\{c_{n}+r_{n}\right\}$ of standard deviation $\sigma$, which will be set to $\sigma=0.0289 \mathrm{RMS}_{0}$, where $\mathrm{RMS}_{0}$ is the RMS of $\left\{c_{n}\right\}$. For both noise-free and noise-corrupted FIDs, the polynomial degree $K$ in $P_{K}^{-} / Q_{K}^{-}$and $(\mathrm{d} / \mathrm{d} \nu)^{m} P_{K}^{-} / Q_{K}^{-}$in the $\mathrm{FPT}^{(-)}$and $\mathrm{dFPT}^{(-)}$, respectively, is set to $K=N / 2=1024$. With this $K$, all the envelopes have fully converged in the $\mathrm{FPT}^{(-)}$and $\mathrm{dFPT}^{(-)}$for each 
of the considered values of $m(1 \leq m \leq 50)$. This was checked by using $N=4096$ with $K=1024,1500,2048$.

\subsubsection{Noiseless reconstructions}

Recall that this sub-section is only on Padé-based signal processing in the $\mathrm{FPT}^{(-)}$ and $\mathrm{dFPT}^{(-)}$. Using the noiseless FID with the metabolites from (37), the extracted three spectral parameters (peak position, width, height) per resonance are graphically illustrated. Therein, the principal focus is on the main spectral region of interest (SRI), $v \in[3.205,3.290] \mathrm{ppm}$, containing 7 metabolites listed in (37). It is predicted by the Lorentzian lineshapes of derivative envelopes that with increasing order $m$ of differentiation, the peak widths shorten, whereas simultaneously and proportionally, the peak heights augment. These features permit extraction of the peak parameters (positions, widths, heights) from any $m$ th order derivative envelope spectrum.

Particularly for real values of complex higher-order derivative spectra, to avoid dealing with an increasing number of negative lobes (dips) per resonance for any $m>0$, we shall focus on the magnitude modes of envelopes. Note, that there is no problem to extract the peak parameters from the magnitude mode. Here, it is only a matter of identifying the correct scaling factor for the magnitude envelope in the case of the $m$ th order $(m>0)$ derivative spectrum to obtain the correct peak parameters of the zeroth-order derivative absorption. For example, the peak width of the zeroth-order ( $m=0)$ magnitude mode $\left|P_{K-1}^{-}(\omega) / Q_{K}^{-}(\omega)\right|$ is wider by a factor of $\sqrt{3}$ relative to the peak width of the corresponding absorption, $\operatorname{Re}\left(P_{K-1}^{-}(\omega) / Q_{K}^{-}(\omega)\right)$.

Figure 1 amply illustrates what has just been said about the relation between the peak widths in $\operatorname{Re}\left(P_{K}^{-} / Q_{K}^{-}\right)$and $\left|P_{K}^{-} / Q_{K}^{-}\right|$. The mechanism which explains the width broadening on panel (d) relative to panel (b) of Fig. 1 is the interference between the absorptive $\operatorname{Re}\left(P_{K}^{-} / Q_{K}^{-}\right)$and dispersive $\operatorname{Im}\left(P_{K}^{-} / Q_{K}^{-}\right)$lineshapes from panels (b) and (c), respectively, in the magnitude envelope, $\left|P_{K}^{-} / Q_{K}^{-}\right|=$ $\left|\operatorname{Re}\left(P_{K}^{-} / Q_{K}^{-}\right)+i \cdot \operatorname{Im}\left(P_{K}^{-} / Q_{K}^{-}\right)\right|$. For completeness, panel (a) shows the lactate, Lac, and alanine, Ala, resonances, with the former being the tallest peak in the entire chemical shift range under study, $v \in[1.30,3.29] \mathrm{ppm}$.

The augmented peak heights with the increasing differentiation order $m$ will not be possible to match with the input data $h_{k}$ from (41). On the other hand, the peak heights for ordinary (non-derivative) and derivative spectra are all in arbitrary units, au. Therefore, introducing a normalization for each differentiation order $m$ separately, the increased peak heights on derivative spectra for $m>0$ can still be plotted on the same graphs with the envelopes for $m=0$. This normalization requires that the Padé-reconstructed spectral envelopes have converged with respect to model order $K$ for both $m=0$ and $m>0$, as presently achieved. In such a case, the mentioned normalization consists of equating maximum values of the magnitude envelopes in the $\mathrm{FPT}^{(-)}$and $\mathrm{dFPT}^{(-)}$. This gives the normalization conditions:

$$
\max \left|\left(\frac{\mathrm{d}}{\mathrm{d} v}\right)^{m} \frac{P_{K}^{-}\left(z^{-1}\right)}{Q_{K}^{-}\left(z^{-1}\right)}\right|=\max \left|\frac{P_{K}^{-}\left(z^{-1}\right)}{Q_{K}^{-}\left(z^{-1}\right)}\right|(m=1,2,3, \ldots)
$$


Non-parametric Estimations of Spectral Lineshapes in MRS: Envelopes for Synthesized Noiseless FIDs

Pade-Reconstructed Full Lines for Three Spectral Modes: Absorption, Dispersion and Magnitude

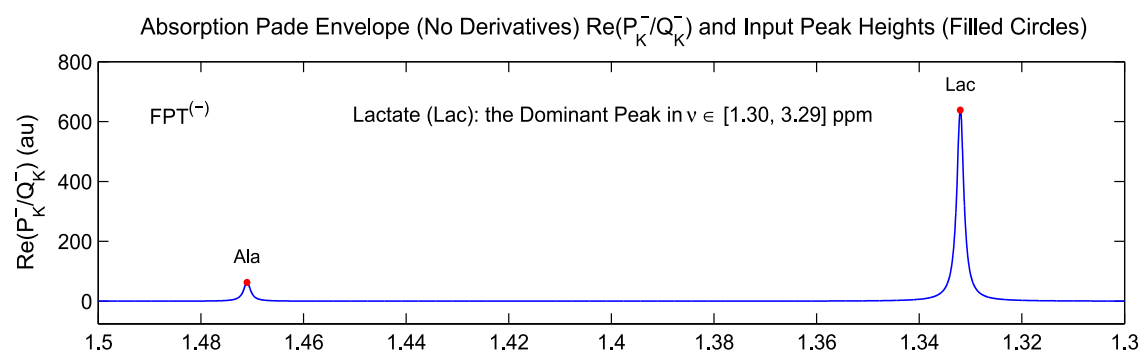

(a) Chemical Shift (ppm)

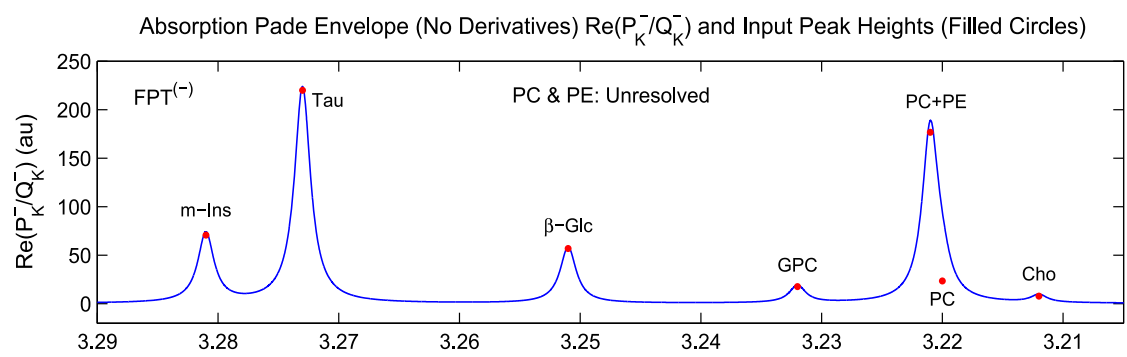

(b) Chemical Shift (ppm)

Dispersion Pade Envelope (No Derivatives) $\operatorname{Im}\left(\mathrm{P}_{\mathrm{K}}^{-} / \mathrm{Q}_{\mathrm{K}}^{-}\right)$and Input Peak Positions (Filled Circles)

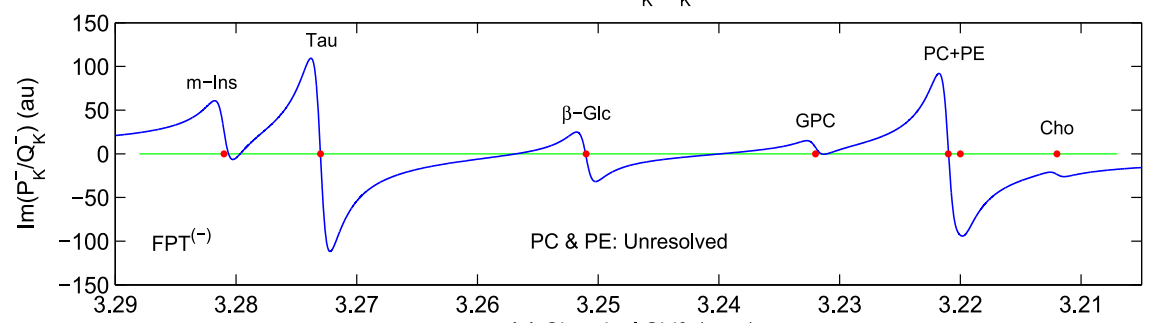

(c) Chemical Shift (ppm)

Magnitude Pade Envelope (No Derivatives) $\left|P_{K}^{-} / Q_{K}^{-}\right|$and Input Peak Heights (Filled Circles)

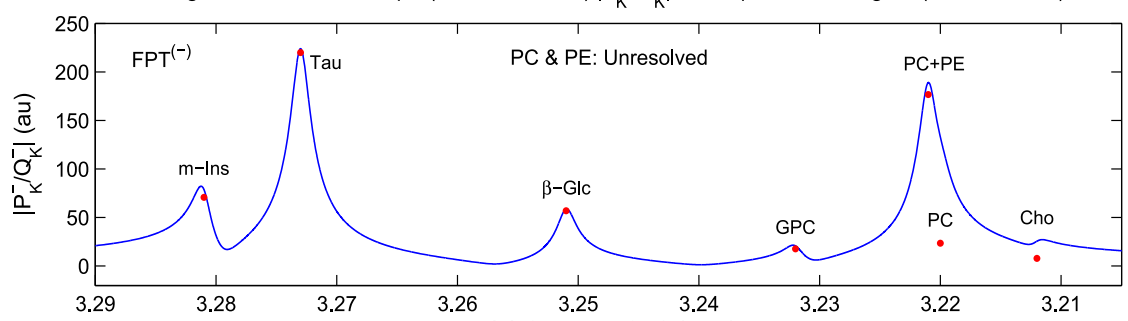

(d) Chemical Shift (ppm)

Fig. 1 Derivative fast Padé transform, $\mathrm{dFPT}^{(-)}$, for a synthesized time signal sampled by using $c_{n}$ from (24) and the spectral parameters (37) according to the FIDs encoded by in vitro MRS from excised human breast cancer tissue, as per Ref. [12]. Two separate narrow bands of chemical shifts (a): $v \in[1.3,1.5] \mathrm{ppm},(\mathbf{b})-$ (d): $v \in[3.205,3.29] \mathrm{ppm}$. Absorption $\operatorname{Re}\left(P_{K}^{-} / Q_{K}^{-}\right)$: (a) and (b), dispersion $\operatorname{Im}\left(P_{K}^{-} / Q_{K}^{-}\right):(\mathbf{c})$, magnitude $\left|P_{K}^{-} / Q_{K}^{-}\right|:(\mathbf{d})$. Circles denote the input data for peak heights $h_{k}$ from (41). (Color online) 
for the chosen range of sweep frequency $\nu$, entrenched in $z^{-1}=\exp (-2 \pi i \tau \nu)$. For the problem under study, normalization (47) occurs at the location of lactate, Lac, from Fig. 1a. Thus, the ratios $\left\{\max \left|(\mathrm{d} / \mathrm{d} \nu)^{m} P_{K}^{-} / Q_{K}^{-}\right|\right\} /\left\{\max \left|P_{K}^{-} / Q_{K}^{-}\right|\right\}$ $(m=1,2,3, \ldots)$ are all equal to unity for the Lac peak, which is, as noted, the tallest in the local spectrum, $v \in[1.30,3.29] \mathrm{ppm}$.

The effect of spectra differentiation becomes noticeable already on the magnitude mode of the 1 st order derivative envelope, $\left|(\mathrm{d} / \mathrm{d} v) P_{K}^{-} / Q_{K}^{-}\right|$, shown in panel (b) of Fig. 2. On this panel, $\left|(\mathrm{d} / \mathrm{d} \nu) P_{K}^{-} / Q_{K}^{-}\right|$is seen as being able to narrow all the peak widths relative to $\left|P_{K}^{-} / Q_{K}^{-}\right|$on Fig. 1d. Such a width narrowing on Fig. $2 \mathrm{~b}$ makes the magnitude $\left|(\mathrm{d} / \mathrm{d} v) P_{K}^{-} / Q_{K}^{-}\right|$very closely approach the absorption lineshape $\operatorname{Re}\left(P_{K}^{-} / Q_{K}^{-}\right)$on panel (a). In other words, the mechanism of the width narrowing by the derivative transform consists of reducing the interference effect. This is the opposite interaction to that in passing from the absorption $\operatorname{Re}\left(P_{K}^{-} / Q_{K}^{-}\right)$and dispersion $\operatorname{Im}\left(P_{K}^{-} / Q_{K}^{-}\right)$to the magnitude $\left|P_{K}^{-} / Q_{K}^{-}\right|$when going from panels (b) and (c) to panel (d) in Fig. 1. The most important difference, albeit relatively small, between panels (a) and (b) of Fig. 2 occurs at the critical chemical shifts 3.220-3.221 ppm, where the tightly overlapped PC and PE peaks reside. Here, on panel (b) for $\left|(\mathrm{d} / \mathrm{d} \nu) P_{K}^{-} / Q_{K}^{-}\right|$, the PE peak is a bit shorter than its counterpart on panel (a). This happens because on panel (b), a part of the preceding PE intensity is taken up by PC whose near-emergence appears as a very slight shoulder on the lower right side of PE. Importantly, on panel (b), the input peak height $h_{\mathrm{PE}}$ from (41) is seen to match the maximum of the magnitude envelope $\left|(\mathrm{d} / \mathrm{d} \nu) P_{K}^{-} / Q_{K}^{-}\right|$at the input chemical shift $3.221 \mathrm{ppm}$ of PE. This is an improvement relative to panels (b) and (d) of Fig. 1. The noticeable interference effect between the absorption (Fig. 1b) and dispersion (Fig. 1c) disturbed the proper peak heights in the magnitude $\left|P_{K}^{-} / Q_{K}^{-}\right|$(Fig. 1d) for several of the resonances. The correct distribution of peak heights is restored on panel (b) of Fig. 2 for $\left|(\mathrm{d} / \mathrm{d} v) P_{K}^{-} / Q_{K}^{-}\right|$for all the metabolites, except for PC.

Also shown on Fig. 2 are the 2 nd and 3rd order derivatives $\left|(\mathrm{d} / \mathrm{d} \nu)^{2} P_{K}^{-} / Q_{K}^{-}\right|$ and $\left|(\mathrm{d} / \mathrm{d} \nu)^{3} P_{K}^{-} / Q_{K}^{-}\right|$on panels (c) and (d), respectively. What had been a barely noticeable shoulder near PC on panel (b) for $\left|(\mathrm{d} / \mathrm{d} \nu) P_{K}^{-} / Q_{K}^{-}\right|$has now become a clearer PC structure in $\left|(\mathrm{d} / \mathrm{d} \nu)^{2} P_{K}^{-} / Q_{K}^{-}\right|$on panel (c). This latter structure is eventually converted into a well delineated PC peak on panel (d). Still, the 3rd order derivative $\left|(\mathrm{d} / \mathrm{d} v)^{3} P_{K}^{-} / Q_{K}^{-}\right|$is not sufficiently high to predict the correct peak height $h_{\mathrm{PC}}$ from (41) for the PC resonance. Moreover, the PC structures on panels (c) and (d) of Fig. 2 are not yet located at the exact input chemical shift, $3.220 \mathrm{ppm}$.

Next, Figs. 3-6 present the higher-order derivatives of spectral envelopes in the magnitude modes. Similarly to Figs. 1 and 2, the reference or control spectrum, the absorption envelope $\operatorname{Re}\left(P_{K}^{-} / Q_{K}^{-}\right)$, is always given in Figs. 3-6 to guide the eye through the systematic and successive process of peak width narrowing with augmenting derivative order $m$ in $\left|(\mathrm{d} / \mathrm{d} \nu)^{m} P_{K}^{-} / Q_{K}^{-}\right|$. The concomitant peak height enlargement with increasing derivative order $m$ in $\left|(\mathrm{d} / \mathrm{d} \nu)^{m} P_{K}^{-} / Q_{K}^{-}\right|$is, however, not to be seen in Figs. 3-6 because of the normalization (47).

Computations of noiseless derivative spectra were carried out for $m=1,2,3, \ldots$, 50 , i.e. with the step $\Delta m=1$, so that $m=1(1) 50$. Here, the number 1 in the parentheses refers to the increment $\Delta m=1$. However, to avoid clutter, only the 
Quantification by Shape Estimation: Synthesized Noiseless FIDs, Input Peak Heights (Filled Circles)

Full Lines (Pade): Absorption and Magnitudes of Envelope Derivatives, $\left|(d / d v)^{m} P_{K}^{-} / Q_{K}^{-}\right| ; m=1,2,3$

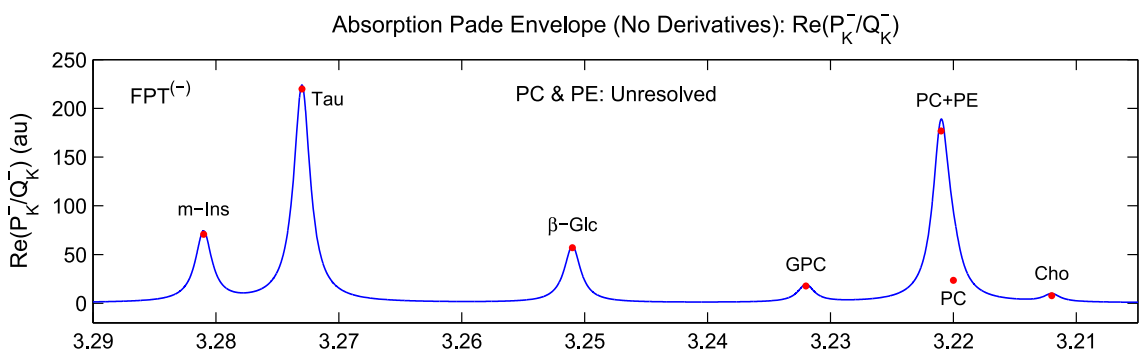

(a) Chemical Shift (ppm)

Magnitude of the 1st Derivative of the Pade Complex Envelope $\left|(d / d v) P_{K}^{-} / Q_{K}^{-}\right|$

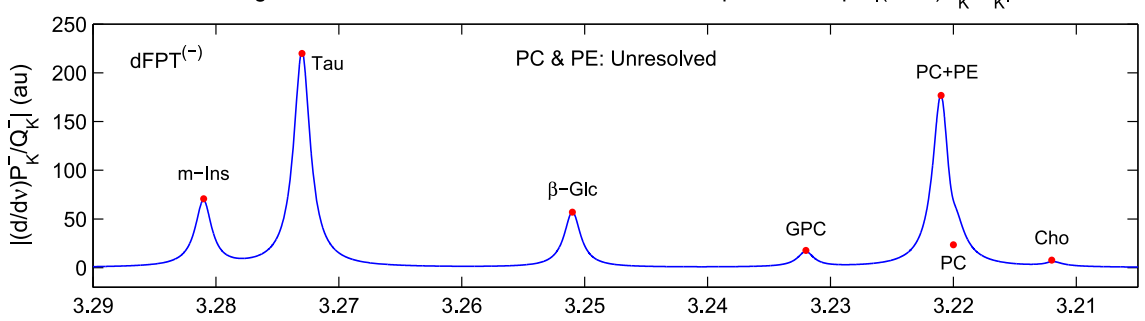

(b) Chemical Shift (ppm)

Magnitude of the 2nd Derivative of the Pade Complex Envelope: $\left|(\mathrm{d} / \mathrm{dv})^{2} \mathrm{P}_{\mathrm{K}}^{-} / \mathrm{Q}_{\mathrm{K}}^{-}\right|$

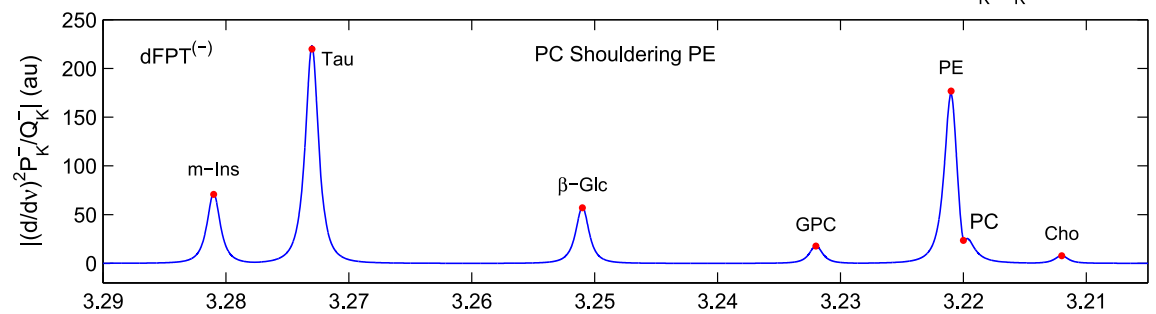

(c) Chemical Shift (ppm)

Magnitude of the 3rd Derivative of the Pade Complex Envelope: $\left|(\mathrm{d} / \mathrm{dv})^{3} \mathrm{P}_{\mathrm{K}}^{-} / \mathrm{Q}_{\mathrm{K}}^{-}\right|$

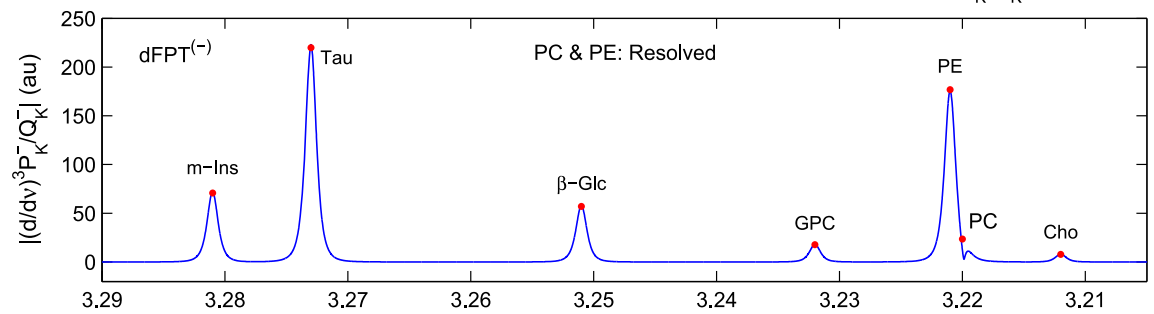

(d) Chemical Shift (ppm)

Fig. 2 Derivative fast Padé transform, $\mathrm{dFPT}^{(-)}$, for a synthesized time signal sampled by using $c_{n}$ from (24) and the spectral parameters (37) according to the FIDs encoded by in vitro MRS from excised human breast cancer tissue, as per Ref. [12]. SRI: $v \in[3.205,3.290] \mathrm{ppm}$. Absorption $\operatorname{Re}\left(P_{K}^{-} / Q_{K}^{-}\right)$: (a), magnitudes $\left|(\mathrm{d} / \mathrm{d} \nu) P_{K}^{-} / Q_{K}^{-}\right|:(\mathbf{b}),\left|(\mathrm{d} / \mathrm{d} v)^{2} P_{K}^{-} / Q_{K}^{-}\right|:(\mathbf{c}),\left|(\mathrm{d} / \mathrm{d} v)^{3} P_{K}^{-} / Q_{K}^{-}\right|:(\mathbf{d})$. Circles denote the input data for peak heights $h_{k}$ from (41). (Color online) 
Quantification by Shape Estimation: Synthesized Noiseless FIDs, Input Peak Heights (Filled Circles) Full Lines (Pade): Absorption and Magnitudes of Envelope Derivatives, $\left|(d / d v)^{m} P_{K^{-}}^{-} / Q_{K}^{-}\right| ; m=6,10,14$

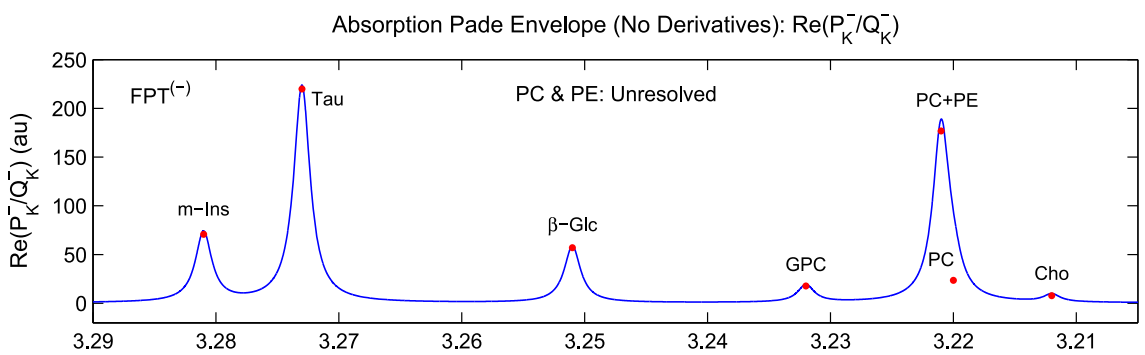

(a) Chemical Shift (ppm)

Magnitude of the 6th Derivative of the Pade Complex Envelope: $\left|(d / d v)^{6} P_{K}^{-} / Q_{K}^{-}\right|$

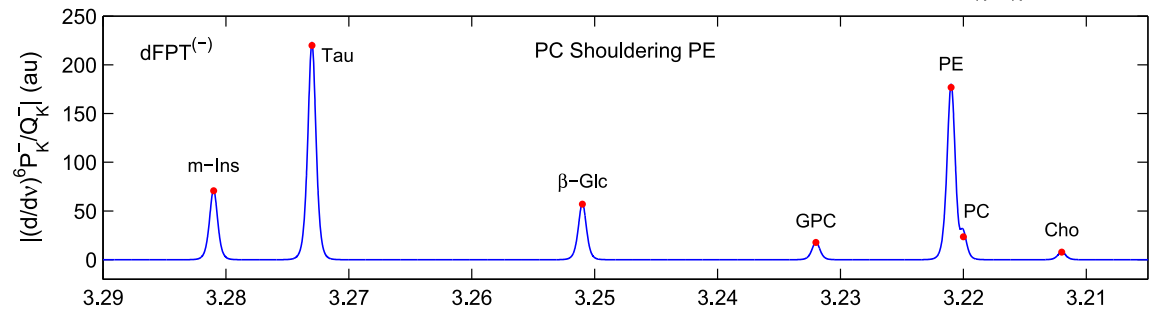

(b) Chemical Shift (ppm)

Magnitude of the 10th Derivative of the Pade Complex Envelope: $\left|(d / d v)^{10} P_{K}^{-} / Q_{K}^{-}\right|$

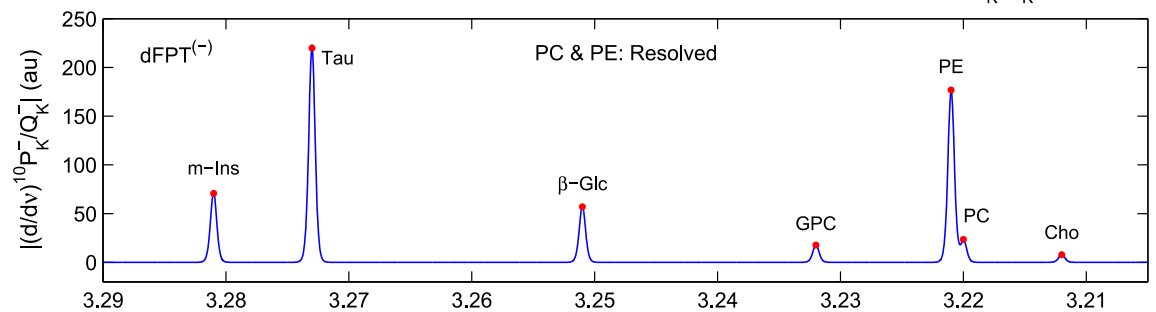

(c) Chemical Shift (ppm)

Magnitude of the 14th Derivative of the Pade Complex Envelope: $\left|(d / d v){ }^{14} P_{K}^{-} / Q_{K}^{-}\right|$

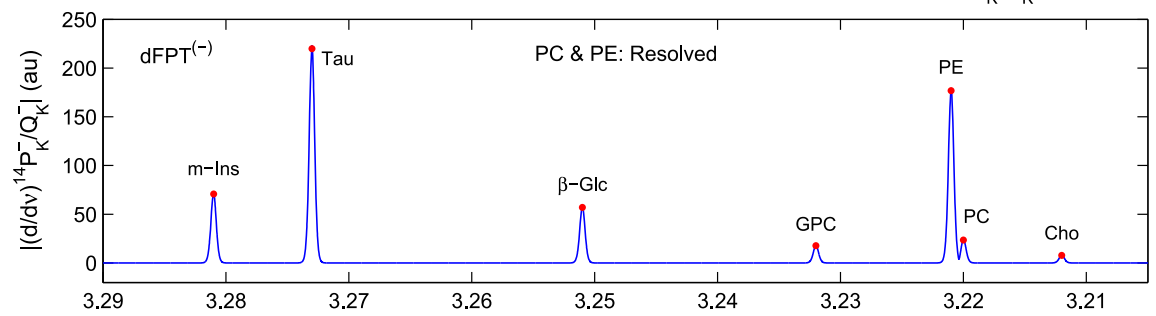

(d) Chemical Shift (ppm)

Fig. 3 Derivative fast Padé transform, $\mathrm{dFPT}^{(-)}$, for a synthesized time signal sampled by using $c_{n}$ from (24) and the spectral parameters (37) according to the FIDs encoded by in vitro MRS from excised human breast cancer tissue, as per Ref. [12]. SRI: $v \in[3.205,3.290] \mathrm{ppm}$. Absorption $\operatorname{Re}\left(P_{K}^{-} / Q_{K}^{-}\right):(\mathbf{a})$, magnitudes $\left|(\mathrm{d} / \mathrm{d} v)^{6} P_{K}^{-} / Q_{K}^{-}\right|:(\mathbf{b}),\left|(\mathrm{d} / \mathrm{d} v)^{10} P_{K}^{-} / Q_{K}^{-}\right|:(\mathbf{c}),\left|(\mathrm{d} / \mathrm{d} v)^{14} P_{K}^{-} / Q_{K}^{-}\right|$: (d). Circles denote the input data for peak heights $h_{k}$ from (41). (Color online) 
Quantification by Shape Estimation: Synthesized Noiseless FIDs, Input Peak Heights (Filled Circles)

Full Lines (Pade): Absorption and Magnitudes of Envelope Derivatives, $\left|(d / d v)^{m} P_{K}^{-} / Q_{K}^{-}\right| ; m=18,22,26$

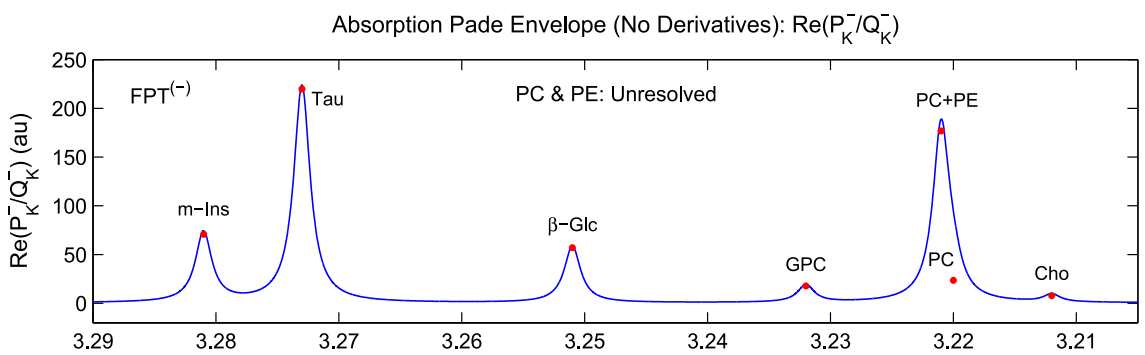

(a) Chemical Shift (ppm)

Magnitude of the 18th Derivative of the Pade Complex Envelope: $\left|(d / d v)^{18} P_{K}^{-} / Q_{K}^{-}\right|$

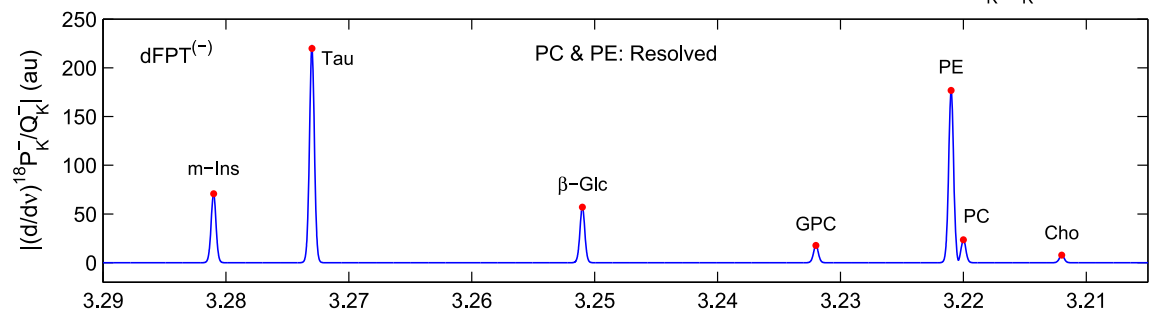

(b) Chemical Shift (ppm)

Magnitude of the 22nd Derivative of the Pade Complex Envelope: $\left|(\mathrm{d} / \mathrm{dv})^{22} \mathrm{P}_{\mathrm{K}^{-}} / \mathrm{Q}_{\mathrm{K}}^{-}\right|$

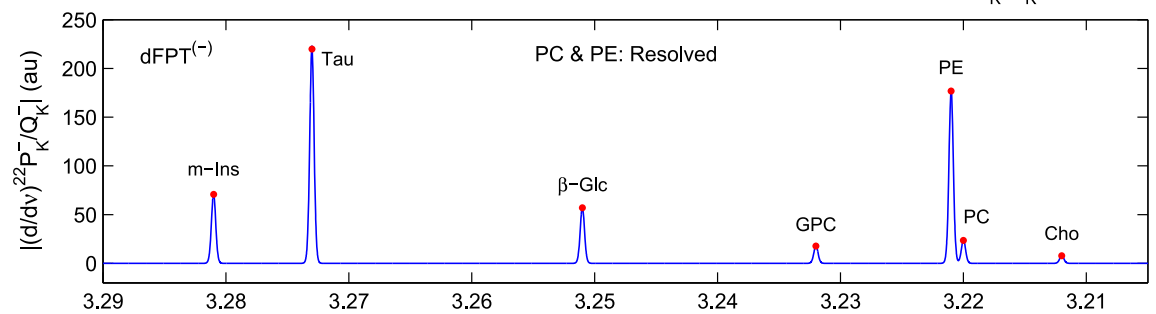

(c) Chemical Shift (ppm)

Magnitude of the 26th Derivative of the Pade Complex Envelope: $\left|(d / d v)^{26} P_{K^{-}} / Q_{K}^{-}\right|$

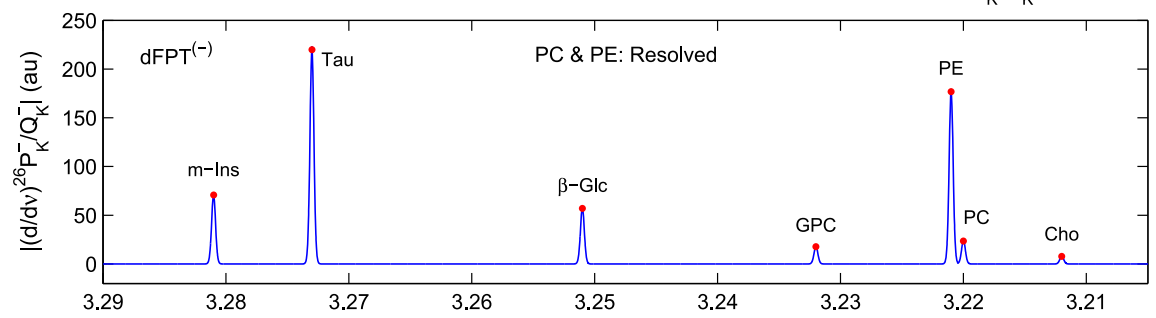

(d) Chemical Shift (ppm)

Fig. 4 Derivative fast Padé transform, $\mathrm{dFPT}^{(-)}$, for a synthesized time signal sampled by using $c_{n}$ from (24) and the spectral parameters (37) according to the FIDs encoded by in vitro MRS from excised human breast cancer tissue, as per Ref. [12]. SRI: $v \in[3.205,3.290] \mathrm{ppm}$. Absorption $\operatorname{Re}\left(P_{K}^{-} / Q_{K}^{-}\right):(\mathbf{a})$, magnitudes $\left|(\mathrm{d} / \mathrm{d} v)^{18} P_{K}^{-} / Q_{K}^{-}\right|:(\mathbf{b}),\left|(\mathrm{d} / \mathrm{d} v)^{22} P_{K}^{-} / Q_{K}^{-}\right|:(\mathbf{c}),\left|(\mathrm{d} / \mathrm{d} v)^{26} P_{K}^{-} / Q_{K}^{-}\right|:(\mathbf{d})$. Circles denote the input data for peak heights $h_{k}$ from (41). (Color online) 
Quantification by Shape Estimation: Synthesized Noiseless FIDs, Input Peak Heights (Filled Circles)

Full Lines (Pade): Absorption and Magnitudes of Envelope Derivatives, $\left|(d / d v)^{m} P_{K}^{-} / Q_{K}^{-}\right| ; m=30,34,38$

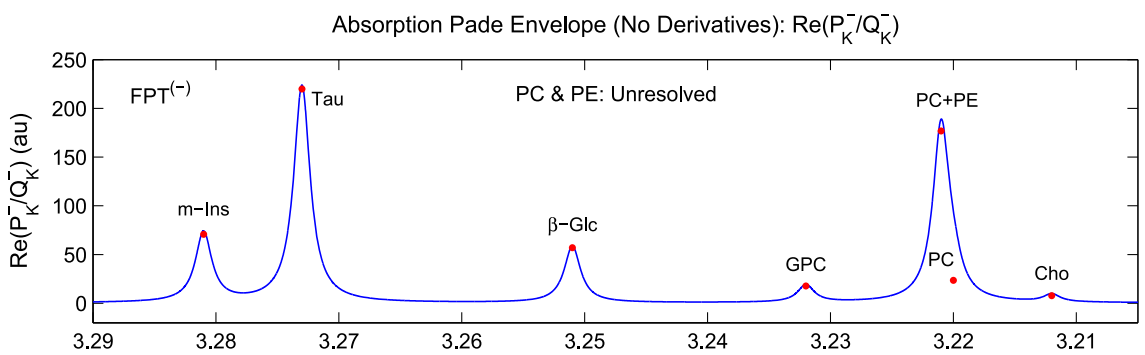

(a) Chemical Shift (ppm)

Magnitude of the 30th Derivative of the Pade Complex Envelope: $\left|(d / d v)^{30} P_{K}^{-} / Q_{K}^{-}\right|$

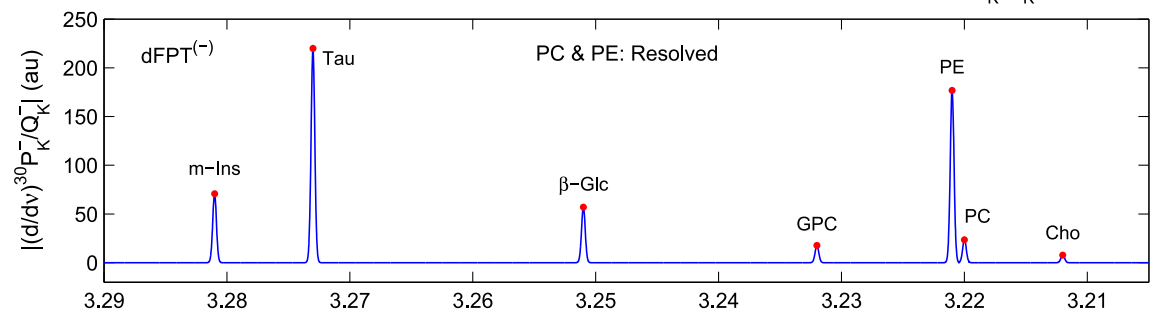

(b) Chemical Shift (ppm)

Magnitude of the 34th Derivative of the Pade Complex Envelope: $\left|(\mathrm{d} / \mathrm{d} v)^{34} \mathrm{P}_{K^{-}} / \mathrm{Q}_{\mathrm{K}}^{-}\right|$

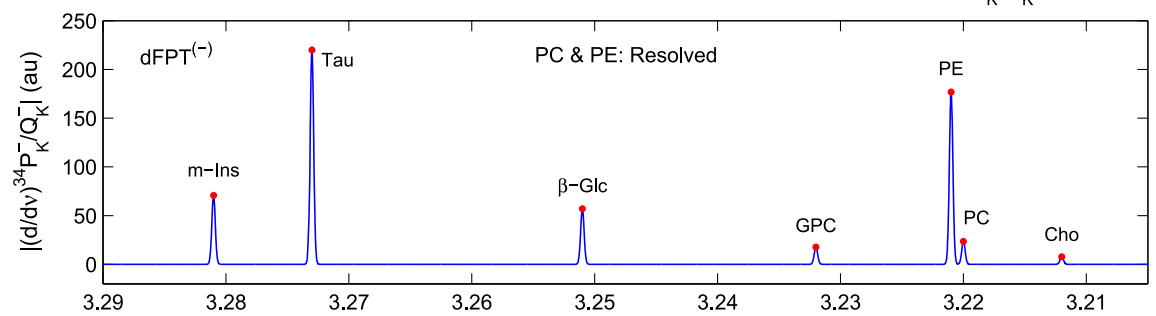

(c) Chemical Shift (ppm)

Magnitude of the 38th Derivative of the Pade Complex Envelope: $\left|(d / d v)^{38} P_{K}^{-} / Q_{K}^{-}\right|$

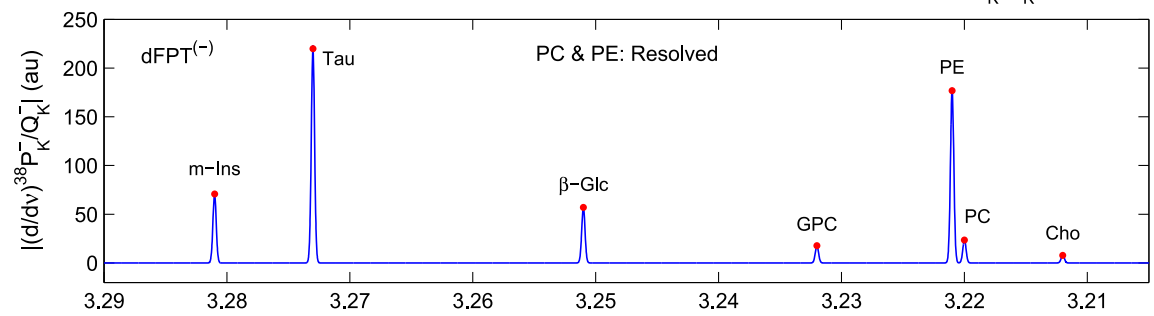

(d) Chemical Shift (ppm)

Fig. 5 Derivative fast Padé transform, $\mathrm{dFPT}^{(-)}$, for a synthesized time signal sampled by using $c_{n}$ from (24) and the spectral parameters (37) according to the FIDs encoded by in vitro MRS from excised human breast cancer tissue, as per Ref. [12]. SRI: $v \in[3.205,3.290] \mathrm{ppm}$. Absorption $\operatorname{Re}\left(P_{K}^{-} / Q_{K}^{-}\right):(\mathbf{a})$, magnitudes $\left|(\mathrm{d} / \mathrm{d} v)^{30} P_{K}^{-} / Q_{K}^{-}\right|:(\mathbf{b}),\left|(\mathrm{d} / \mathrm{d} v)^{34} P_{K}^{-} / Q_{K}^{-}\right|:(\mathbf{c}),\left|(\mathrm{d} / \mathrm{d} v)^{38} P_{K}^{-} / Q_{K}^{-}\right|:(\mathbf{d})$. Circles denote the input data for peak heights $h_{k}$ from (41). (Color online) 
Quantification by Shape Estimation: Synthesized Noiseless FIDs, Input Peak Heights (Filled Circles)

Full Lines (Pade): Absorption and Magnitudes of Envelope Derivatives, $\left|(d / d v)^{m} P_{K}^{-} / Q_{K}^{-}\right| ; m=42,46,50$

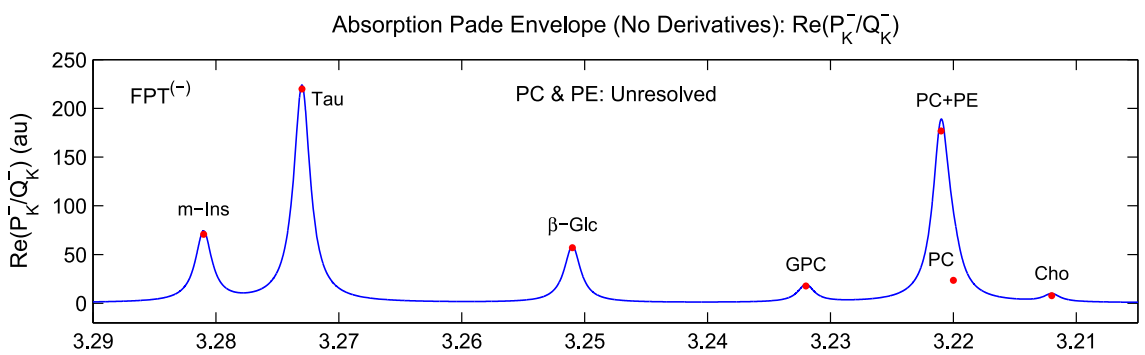

(a) Chemical Shift (ppm)

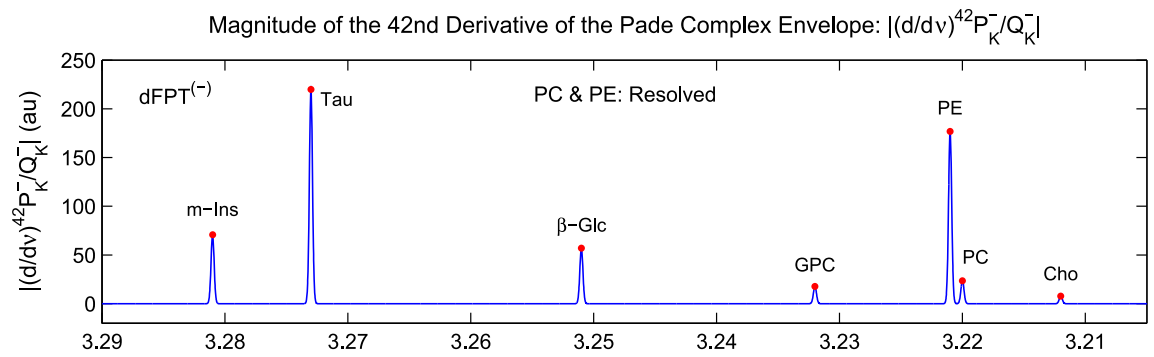

(b) Chemical Shift (ppm)

Magnitude of the 46th Derivative of the Pade Complex Envelope: $\left|(d / d v)^{46} P_{K}^{-} / Q_{K}^{-}\right|$

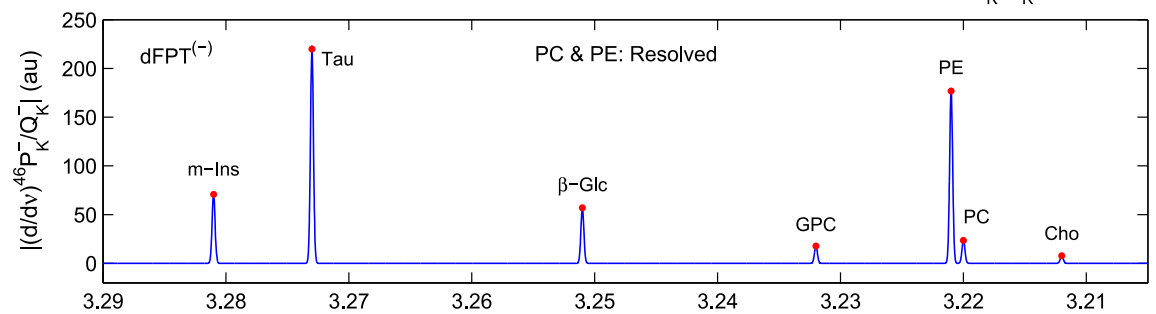

(c) Chemical Shift (ppm)

Magnitude of the 50th Derivative of the Pade Complex Envelope: $\left|(\mathrm{d} / \mathrm{dv})^{50} \mathrm{P}_{\mathrm{K}}^{-} / \mathrm{Q}_{\mathrm{K}}^{-}\right|$

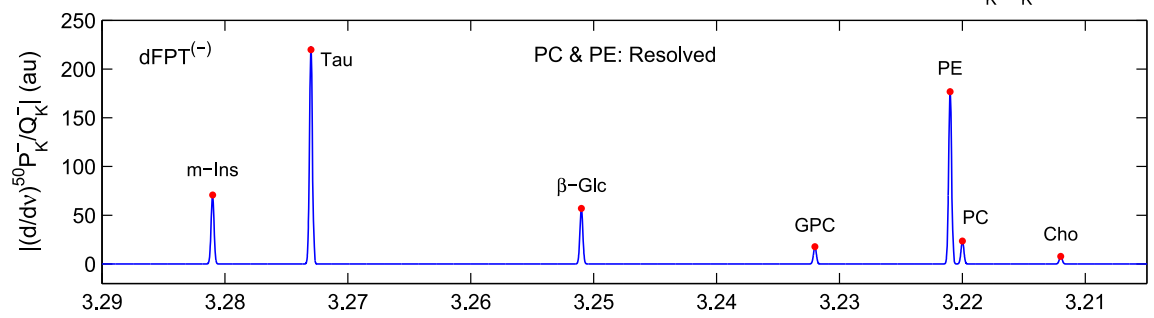

(d) Chemical Shift (ppm)

Fig. 6 Derivative fast Padé transform, $\mathrm{dFPT}^{(-)}$, for a synthesized time signal sampled by using $c_{n}$ from (24) and the spectral parameters (37) according to the FIDs encoded by in vitro MRS from excised human breast cancer tissue, as per Ref. [12]. SRI: $v \in[3.205,3.290] \mathrm{ppm}$. Absorption $\operatorname{Re}\left(P_{K}^{-} / Q_{K}^{-}\right)$: (a), magnitudes $\left|(\mathrm{d} / \mathrm{d} v)^{42} P_{K}^{-} / Q_{K}^{-}\right|:(\mathbf{b}),\left|(\mathrm{d} / \mathrm{d} v)^{46} P_{K}^{-} / Q_{K}^{-}\right|:(\mathbf{c}),\left|(\mathrm{d} / \mathrm{d} v)^{50} P_{K}^{-} / Q_{K}^{-}\right|:(\mathbf{d})$. Circles denote the input data for peak heights $h_{k}$ from (41). (Color online) 
results with a larger step for $\Delta m$ are presented in Figs. 3-6. Therein, we display the derivative envelopes $\left|(\mathrm{d} / \mathrm{d} \nu)^{m} P_{K}^{-} / Q_{K}^{-}\right|$for $m=6(4) 50$, i.e. with $\Delta m=4$.

Already on Fig. 3, it is seen that the 10th and the 14th derivatives on panels (c) and (d) have all the correct peak heights, including $h_{\mathrm{PC}}$ and $h_{\mathrm{PE}}$ for the PC and PE resonances, respectively. Still, only $m=14$ on panel (d) exhibits well separated PC and PE peaks almost all the way down to the baseline, which is the abscissa itself for these noiseless data. Even though the peak height for the PC resonance is correct for $m=10$ on panel (c), the splitting between PC and PE is far from the baseline. The 6th derivative on panel (b) has not yet reached the correct peak height for the PC resonance which, as a shoulder, is not separated from PE.

Figures 4-6, viewed together, display the full converging effect of the differentiation transform in the normalized $\mathrm{dFPT}^{(-)}$. We say converging with respect to $m$, because the pattern of recovering the input peak heights $h_{k}(1 \leq k \leq 9)$ is uniformly maintained for each increasing value of the derivative order $m$ in Fig. 4 ( $m=18,22,26)$, Fig. $5(m=30,34,38)$ and Fig. $6(m=42,46,50)$. The effect of differentiation has reached the saturation level, manifested by the steady configuration (distribution) of all the well separated metabolites. This includes the critical region 3.220-3.221 ppm containing the PC and PE peaks. The two latter overlapping resonances are extremely well delineated on Figs. 4-6, with both of their baselines descending nearly or completely to the zero value of the ordinates (i.e. merged into the abscissae). The maintained stability of the PC peak in the normalized Padé-generated high-order derivative spectral envelopes from Figs. 4-6, is important relative to some oscillations in this regard for the lower-order derivative envelopes $(2 \leq m \leq 10)$ in Figs. 2 and 3 .

Overall, Figs. 1-6 permit us to draw the following general conclusion. The differentiation operator $(\mathrm{d} / \mathrm{d} \nu)^{m}$ in the normalized magnitude mode of the derivative spectral envelopes $\left|(\mathrm{d} / \mathrm{d} v)^{m} P_{K}^{-} / Q_{K}^{-}\right|$systematically improves the reconstructions with increasing order $m(m=1,2, \ldots)$. Improvement is both qualitative (straightening and perfecting the symmetry of the bell-shaped spectral lines) and quantitative (precision in extracting the peak positions, widths and heights). In the normalized spectra $\left|(\mathrm{d} / \mathrm{d} \nu)^{m} P_{K}^{-} / Q_{K}^{-}\right|$, the peak locations and peak heights are steadily maintained for increasing $m$. Stated in the language of signal processing, the differentiation transforms in the $\mathrm{dFPT}^{(-)}$, particularly those of higher orders $m$ of derivatives, dramatically improve the resolution of the spectral envelopes relative to the zeroth-order counterparts $(m=0)$ in the $\operatorname{FPT}^{(-)}$.

For increased $m$, in the same normalized spectra of the magnitude mode $\left|(\mathrm{d} / \mathrm{d} \nu)^{m} P_{K}^{-} / Q_{K}^{-}\right|$, the peak widths are systematically narrowed by the pertinent identifiable scaling factors (available as the analytical expressions) that make possible the exact reconstructions of the input peak widths, proportional to $\operatorname{Im}\left(v_{k}\right)$. Therefore, the derivative spectral envelopes, $\left|(\mathrm{d} / \mathrm{d} \nu)^{m} P_{K}^{-} / Q_{K}^{-}\right|$, especially for higher order $m$, can, in principle, separate any closely overlapped peaks and, most importantly, exactly quantify them. Crucially, as seen in Figs. 5 and 6, the tails of all the derivative resonance lineshapes are entirely embedded in the chemical shift axis. This shows that the differential operator in $\left|(\mathrm{d} / \mathrm{d} \nu)^{m} P_{K}^{-} / Q_{K}^{-}\right|$, no matter how high $m$ is taken, introduces no noise, random or any other kind for that matter (from e.g. round-off errors in numerical computations with finite arithmetics). This is an indication that the $\mathrm{dFPT}^{(-)}$ could also perform well for noisy FIDs to be treated in Sect. 4.2.2. 
The extremely narrow symmetric Lorentzian peaks (from their tips to bottoms) in the magnitudes of the $m$ th derivative complex envelopes for sufficiently high differentiation order $m$, solve yet another practical problem routinely encountered with the corresponding lineshapes for $m=0$. This problem consists of long, extended Lorentzian tails, that additionally mask adjacent and even distant spectral structures of potentially informative content. Extended resonance tails are present in all lineshapes, not only of the Lorentzian profile. One of the methods used for computation of general profile areas is integration by numerical quadratures, as often done in MRS, and this was also the case with the study [12] by reference to which we synthesized the present FIDs. For this purpose, the upper and lower integration limits around a given peak need to be fixed. This evidently becomes a matter of subjectivity. Such a circumstance invalidates the estimates of the peak areas. Consequently, the resulting estimates of the abundance or concentrations of substances (that are metabolites in MRS) will be biased, at best. The high-order derivative envelopes circumvent this obstacle altogether and exactly determine the peak areas, thus yielding the correct concentrations.

As per our analysis of the $\mathrm{dFPT}^{(-)}$in the case of Figs. 1-6 for the SRI, $v \in$ $[3.205,3.290] \mathrm{ppm}$, the most intriguing and clinically relevant finding is in the very narrow region 3.220-3.221 ppm with the closely overlapped PC and PE resonances. Recall that the diagnostic significance of phosphocholine, PC, is in its role as a recognized cancer biomarker. It is, therefore, warranted to zoom into a narrow SRI around PC and PE such as $v \in[3.219,3.222] \mathrm{ppm}$. With this SRI we shall focus the subsequent analysis and illustrations from the $\mathrm{dFPT}^{(-)}$exclusively on the PC and PE peaks for the case of noiseless time signals. This is done in Figs. 7-9 where a similar pattern in consecutiveness of increasing the derivative order $m$ is followed as that in Figs. 3-6. Namely, we begin with smaller differentiation order $m$ in Fig. 7 and proceed further with higher values of $m$ in Figs. 8 and 9. Specifically, Fig. 7 shows real-valued spectra $\operatorname{Re}\left((\mathrm{d} / \mathrm{d} \nu)^{m} P_{K}^{-} / Q_{K}^{-}\right)$and the corresponding magnitudes $\left|(\mathrm{d} / \mathrm{d} \nu)^{m} P_{K}^{-} / Q_{K}^{-}\right|$for $0 \leq m \leq 2$. The former and the latter envelopes are respectively seen on the left column (without normalization) and right column (with normalization) of Fig. 7. The zeroth-order derivative spectrum $(m=0)$ on panel (a) is the standard absorption envelope, $\operatorname{Re}\left(P_{K}^{-} / Q_{K}^{-}\right)$. Its magnitude counterpart, $\left|P_{K}^{-} / Q_{K}^{-}\right|$, is on panel (d). As to the prospect of separating the PC and PE peaks, the situation is initially worsened when passing from the absorption to the magnitude modes for $m=0$. This is seen by having the full line in panel (d) further away from the filled circle for the input peak height of PC than in panel (a). Such an occurrence is caused by the broadening of the peak width in the magnitude mode for $m=0$ relative to the absorption mode. As discussed, this occurs with non-derivative envelopes because of the interference of the absorptive and dispersive lineshapes in (d). Overall, neither the absorption nor the magnitude modes on panels (a) and (d), respectively, give any hint of the existence of the PC resonance. In particular, no curvature change from a smooth, continuous decrease, is noticeable in the full curves anywhere downstream of the PE peak in either panel (a) or (d).

However, this discouraging situation with the non-derivative magnitude spectrum $(m=0)$ on panel $(d)$, is quickly ameliorated on panels (e) and (f) for the asso- 
Quantification by Shape Estimation: Synthesized Noiseless FIDs, Input Peak Heights (Filled Circles)

Full Lines (Pade): Real Parts and Magnitudes of Envelope $P_{K}^{-} / Q_{K}^{-}$and of $(d / d v)^{m} P_{K}^{-} / Q_{K}^{-} ; m=1,2$

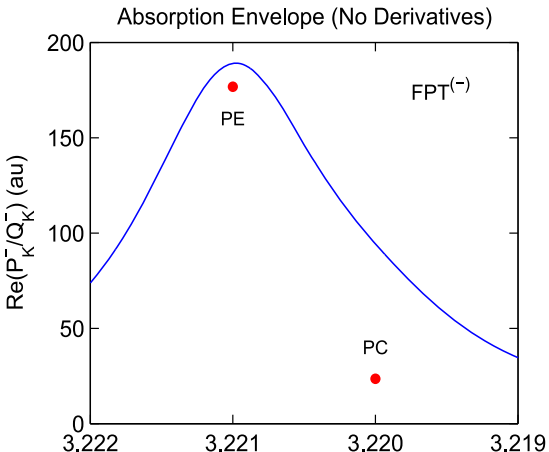

(a) Chemical Shift (ppm)

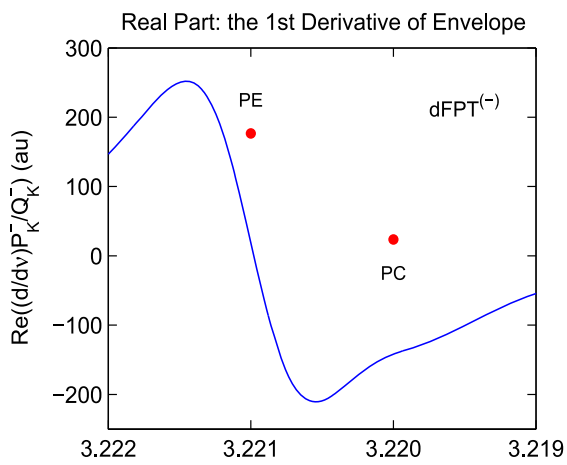

(b) Chemical Shift (ppm)

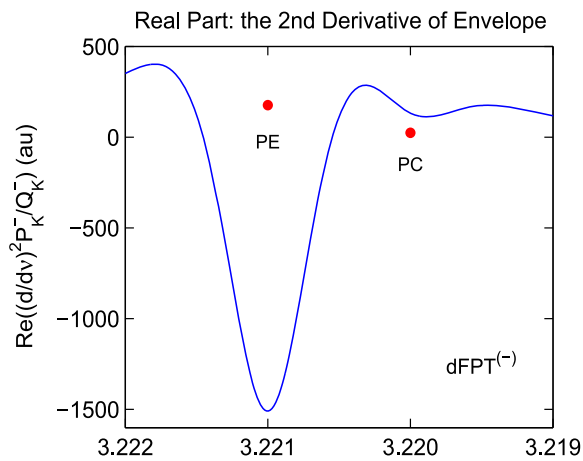

(c) Chemical Shift (ppm)

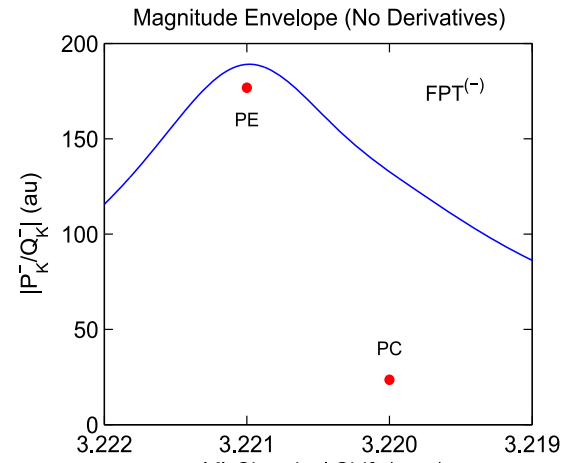

(d) Chemical Shift (ppm)

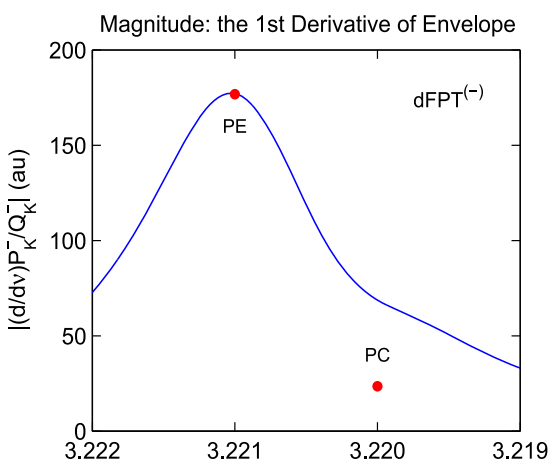

(e) Chemical Shift (ppm)

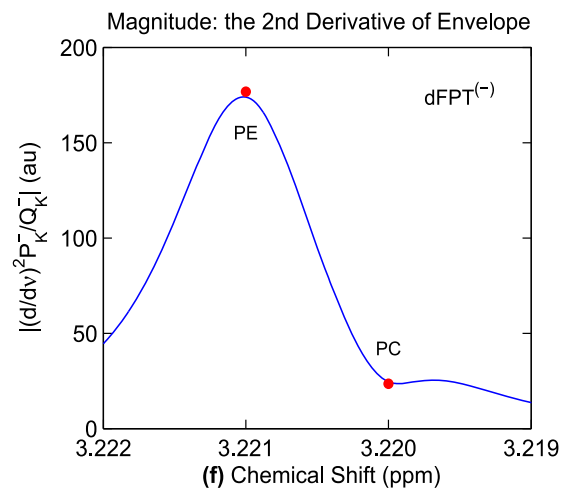

Fig. 7 Derivative fast Padé transform, $\mathrm{dFPT}^{(-)}$, for a synthesized time signal sampled by using $c_{n}$ from (24) and the spectral parameters (37) according to the FIDs encoded by in vitro MRS from excised human breast cancer tissue, as per Ref. [12]. SRI: $v \in[3.219,3.222] \mathrm{ppm}$. Real parts $\operatorname{Re}\left(P_{K}^{-} / Q_{K}^{-}\right)$: (a), $\operatorname{Re}\left((\mathrm{d} / \mathrm{d} v) P_{K}^{-} / Q_{K}^{-}\right)$: (b), $\operatorname{Re}\left((\mathrm{d} / \mathrm{d} v)^{2} P_{K}^{-} / Q_{K}^{-}\right)$: (c). Magnitudes $\left|P_{K}^{-} / Q_{K}^{-}\right|$: (d), $\left|(\mathrm{d} / \mathrm{d} \nu) P_{K}^{-} / Q_{K}^{-}\right|$: (e), $\left|(\mathrm{d} / \mathrm{d} v)^{2} P_{K}^{-} / Q_{K}^{-}\right|$: (f). Circles denote the input data for peak heights $h_{k}$ from (41). (Color online) 
Quantification by Shape Estimation: Synthesized Noiseless FIDs, Input Peak Heights (Filled Circles)

Full Lines (Pade): Magnitude Mode of Envelope Derivatives, $\left|(d / d v)^{m} P_{K}^{-} / Q_{K}^{-}\right| ; m=6$ (4) 26

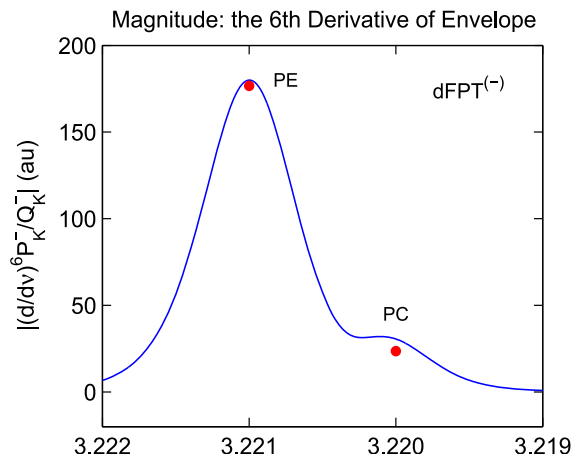

(a) Chemical Shift (ppm)

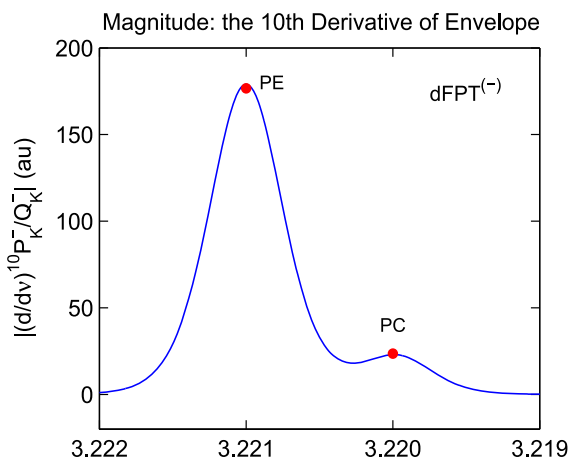

(b) Chemical Shift (ppm)

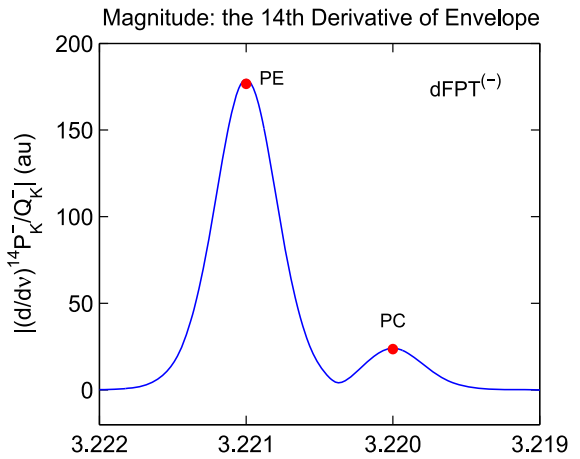

(c) Chemical Shift (ppm)

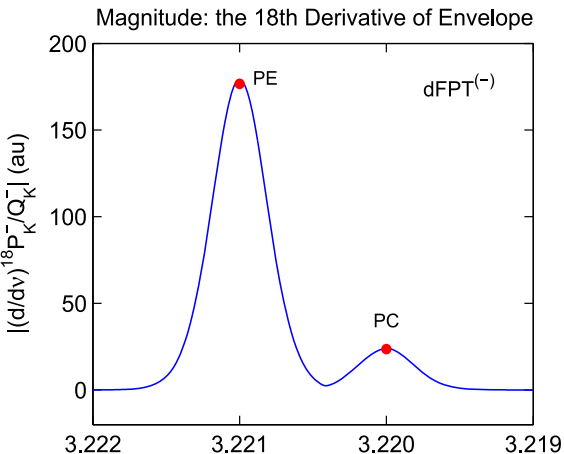

(d) Chemical Shift (ppm)

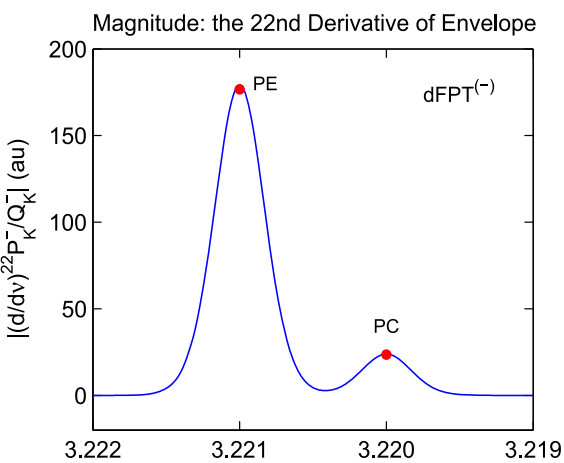

(e) Chemical Shift (ppm)

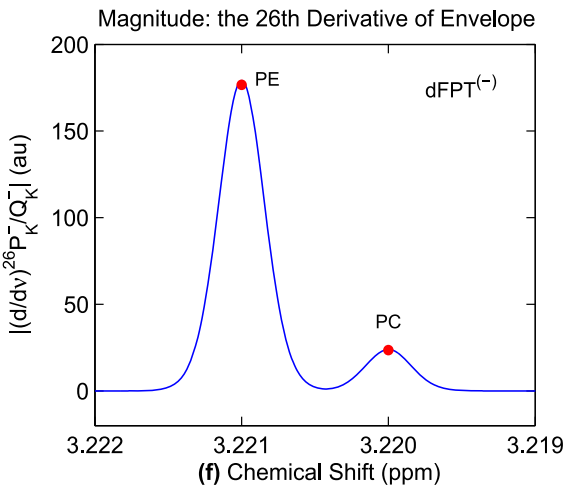

Fig. 8 Derivative fast Padé transform, $\mathrm{dFPT}^{(-)}$, for a synthesized time signal sampled by using $c_{n}$ from (24) and the spectral parameters (37) according to the FIDs encoded by in vitro MRS from excised human breast cancer tissue, as per Ref. [12]. SRI: $v \in[3.219,3.222] \mathrm{ppm}$. Magnitudes $\left|(\mathrm{d} / \mathrm{d} v)^{6} P_{K}^{-} / Q_{K}^{-}\right|$: (a), $\left|(\mathrm{d} / \mathrm{d} v)^{10} P_{K}^{-} / Q_{K}^{-}\right|:(\mathbf{b}),\left|(\mathrm{d} / \mathrm{d} v)^{14} P_{K}^{-} / Q_{K}^{-}\right|:(\mathbf{c}),\left|(\mathrm{d} / \mathrm{d} \nu)^{18} P_{K}^{-} / Q_{K}^{-}\right|:(\mathbf{d}),\left|(\mathrm{d} / \mathrm{d} \nu)^{22} P_{K}^{-} / Q_{K}^{-}\right|:(\mathbf{e})$, $\left|(\mathrm{d} / \mathrm{d} v)^{26} P_{K}^{-} / Q_{K}^{-}\right|:(\mathbf{f})$. Circles denote the input data for peak heights $h_{k}$ from (41). (Color online) 
Quantification by Shape Estimation: Synthesized Noiseless FIDs, Input Peak Heights (Filled Circles) Full Lines (Pade): Magnitude Mode of Envelope Derivatives, $\left|(d / d v)^{m} P_{K}^{-} / Q_{K}^{-}\right| ; m=30(4) 50$

Magnitude: the 30th Derivative of Envelope

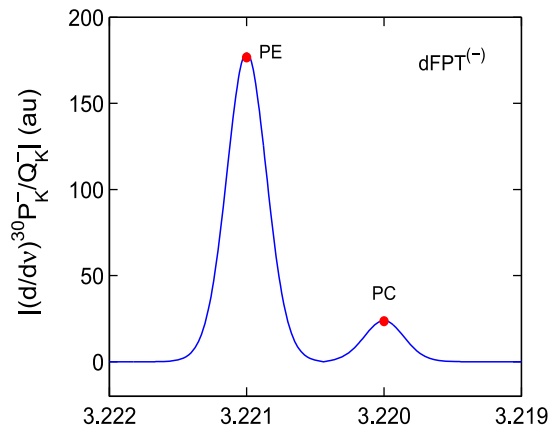

(a) Chemical Shift (ppm)

Magnitude: the 34th Derivative of Envelope

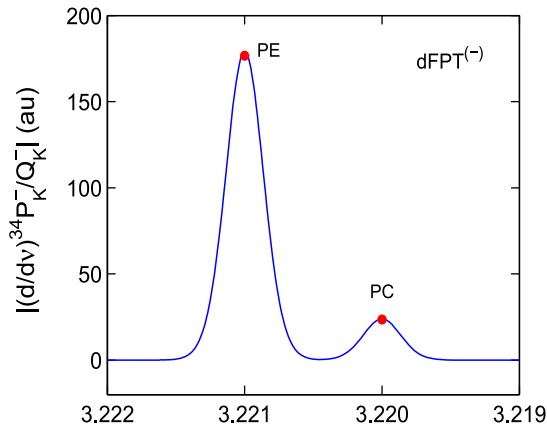

(b) Chemical Shift (ppm)

Magnitude: the 38th Derivative of Envelope

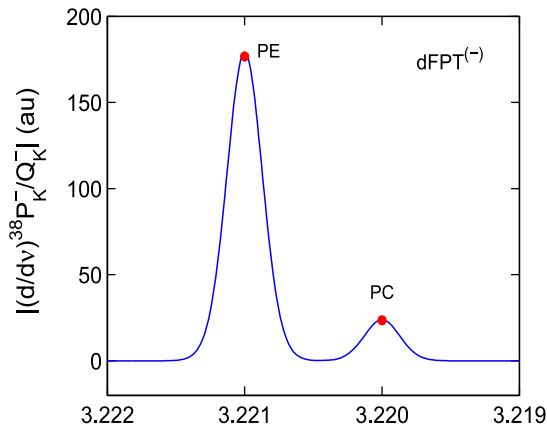

(c) Chemical Shift (ppm)

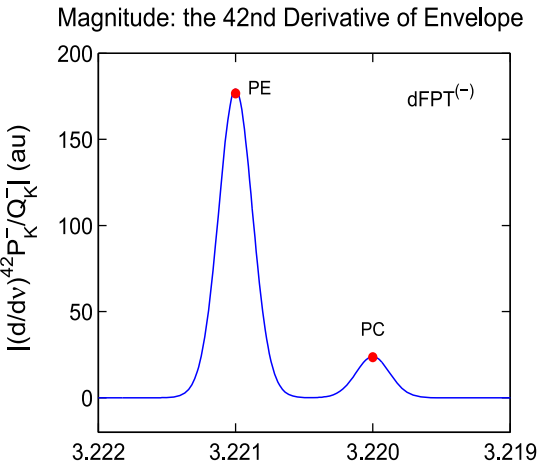

(d) Chemical Shift (ppm)

Magnitude: the 46th Derivative of Envelope

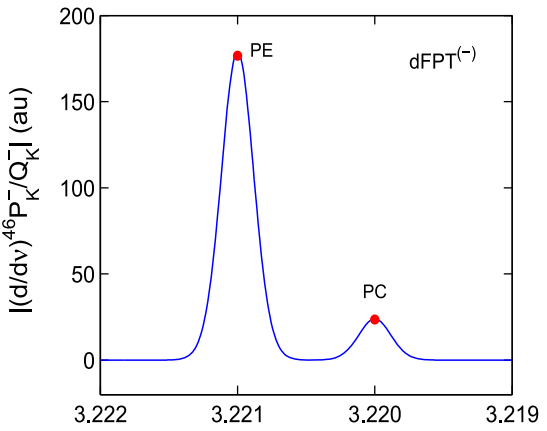

(e) Chemical Shift (ppm)

Magnitude: the 50th Derivative of Envelope

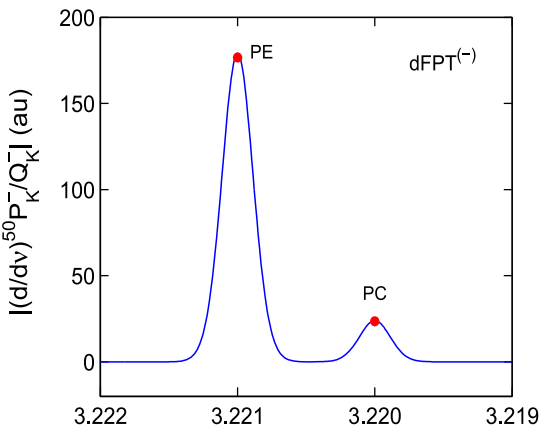

(f) Chemical Shift (ppm)

Fig. 9 Derivative fast Padé transform, $\mathrm{dFPT}^{(-)}$, for a synthesized time signal sampled by using $c_{n}$ from (24) and the spectral parameters (37) according to the FIDs encoded by in vitro MRS from excised human breast cancer tissue, as per Ref. [12]. SRI: $v \in[3.219,3.222] \mathrm{ppm}$. Magnitudes: $\left|(\mathrm{d} / \mathrm{d} \nu)^{30} P_{K}^{-} / Q_{K}^{-}\right|$: (a), $\left|(\mathrm{d} / \mathrm{d} \nu)^{34} P_{K}^{-} / Q_{K}^{-}\right|:(\mathbf{b}),\left|(\mathrm{d} / \mathrm{d} \nu)^{38} P_{K}^{-} / Q_{K}^{-}\right|:(\mathbf{c}),\left|(\mathrm{d} / \mathrm{d} \nu)^{42} P_{K}^{-} / Q_{K}^{-}\right|:(\mathbf{d}),\left|(\mathrm{d} / \mathrm{d} \nu)^{46} P_{K}^{-} / Q_{K}^{-}\right|:(\mathbf{e})$, $\left|(\mathrm{d} / \mathrm{d} \nu)^{50} P_{K}^{-} / Q_{K}^{-}\right|:(\mathbf{f})$. Circles denote the input data for peak heights $h_{k}$ from (41). (Color online) 
ciated 1st $(m=1)$ and the 2 nd $(m=2)$ derivative envelopes $\left|(\mathrm{d} / \mathrm{d} v) P_{K}^{-} / Q_{K}^{-}\right|$ and $\left|(\mathrm{d} / \mathrm{d} v)^{2} P_{K}^{-} / Q_{K}^{-}\right|$, respectively. Thus, on panel (e), an evident slope change is observed in the full curve at the level of the peak height of PC. This indirect indication of the existence of the underlying PC structure in $\left|(\mathrm{d} / \mathrm{d} v) P_{K}^{-} / Q_{K}^{-}\right|$on panel (e) becomes even more suggestive on panel (f) for $\left|(\mathrm{d} / \mathrm{d} v)^{2} P_{K}^{-} / Q_{K}^{-}\right|$. Therein, for the first time, the full curve touches the filled circle for the input peak height of the PC resonance. However, only a dip appears instead of a peak at the input chemical shift for PC. Specifically, an indication of a broad peak is seen downstream, slightly away from the correct chemical shift $3.220 \mathrm{ppm}$ for PC. By comparison with panel (f), the real-valued envelope $\operatorname{Re}\left((\mathrm{d} / \mathrm{d} \nu)^{2} P_{K}^{-} / Q_{K}^{-}\right)$on panel (c) exhibits a completely different pattern. Both the PE and PC resonances are characterized by their minimae on panel (c). While the PE minimum is quite symmetrical and, thus, reasonably well delineated, the other minimum is only suggestive, at best, of the existence of PC. As to the 1 st derivative real-valued spectrum $\operatorname{Re}\left((\mathrm{d} / \mathrm{d} v) P_{K}^{-} / Q_{K}^{-}\right)$on panel (b), its lineshape is of an asymmetric dispersion type. Asymmetry appears as a slight curvature change in the full curve around the PC position, $3.220 \mathrm{ppm}$. This corresponds to the change in slope of the associated positive-definite spectrum $\left|(\mathrm{d} / \mathrm{d} v) P_{K}^{-} / Q_{K}^{-}\right|$on panel (e).

Figures 8 and 9 are also for the narrow band as in Fig. 7, but they deal only with the magnitudes of the Padé derivative spectra normalized according to (47) for the extended SRI: $v \in[1.30,3.29] \mathrm{ppm}$. Figure 8 presents the derivative spectral envelopes in the magnitude modes, $\left|(\mathrm{d} / \mathrm{d} v)^{m} P_{K}^{-} / Q_{K}^{-}\right|$, for $6 \leq m \leq 26$, with $m=$ $2+\Delta m, \ldots, 22+\Delta m$, where $\Delta m=4$. A gradual emergence of the PC peak is seen by proceeding from panel (a) to panel (f) on Fig. 8. On panel (a) for $m=6$, merely a bump is observed surrounding the peak tip of PC. Therein, the peak height of the $\mathrm{PE}$ resonance is very slightly overestimated. A broad, small, asymmetric maximum is present on panel (b) for $m=10$, and therein the full curve of $\left|(\mathrm{d} / \mathrm{d} v)^{10} P_{K}^{-} / Q_{K}^{-}\right|$ coincides with the filled circle for the input data of the peak height of PC. The peak height of PE is also seen to be correctly predicted by the 10th derivative envelope. From panel (c) up to panel (f), the PC peak becomes completely clear, symmetrical and with the correct peak height, $h_{\mathrm{PC}}$. The same applies to $h_{\mathrm{PE}}$ for the dominant PE resonance. By progressing from panel (a) to panel (f), the resonance widths of both $\mathrm{PE}$ and PC are systematically narrowed. Further, the peak-to-peak vertical distance remains constant on panels (c)-(f). The peak heights $h_{\mathrm{PC}}$ and $h_{\mathrm{PE}}$ on panels (b)-(f) are correctly placed at the input chemical shifts.

Figure 9 displays the highest-order derivative spectral envelopes $\left|(\mathrm{d} / \mathrm{d} v)^{m} P_{K}^{-} / Q_{K}^{-}\right|$ for $m=30$ (4) 50 on panels (a)-(f), i.e. for $m=26+\Delta m, \ldots, 46+\Delta m$, with $\Delta m=4$. Here, strikingly, the peak width narrowing has proceeded to such an extent that the baseline between the PC and PE peaks becomes flattened to the level of the pure chemical shift axis. This is best seen by comparing panels (a) and (f) for $m=30$ and $m=50$, respectively. On panel (a), there is a rolling valley between PC and PE. This valley becomes mainly a straight line on panel (f) for a considerable portion of the PC-PE distance on the chemical shift axis. Moreover, the PE-PC peak height ratio has completely stabilized, to the exact ratio 7.5 given by the corresponding input data from (41). Likewise, the PE-PC peak width ratio has also steadily maintained the exact value equal to 1, from panel (a) to panel (f), i.e. throughout Fig. 9. This is 
fully in accordance with the input data (41), where all the full widths at half maximae $\{\mathrm{FWHM}\}_{k}$ are the same, since $\operatorname{Im}\left(v_{k}\right)=0.0008 \mathrm{ppm}(1 \leq k \leq 9)$ as per (37), so that $\{\mathrm{FWHM}\}_{k} /\{\mathrm{FWHM}\}_{k^{\prime}}=1$ for any two $k$ and $k^{\prime}\left(1 \leq k \leq 9,1 \leq k^{\prime} \leq 9, k^{\prime} \neq k\right)$. Also, throughout Fig. 9 the input chemical shifts 3.220 and $3.221 \mathrm{ppm}$ are seen to exactly match the positions (locations) of the Padé-reconstructed PC and PE peaks, respectively.

Overall, in the $\mathrm{dFPT}^{(-)}$, for the higher-order derivative envelopes, the reconstructed peak positions $\operatorname{Re}\left(v_{k}\right)$ do not alter, whereas simultaneously, the peak widths $\operatorname{Im}\left(v_{k}\right)$ decrease and the peak heights $h_{k}$ increase. These two latter trends are mutually compensating in the sense that their product $h_{k} \operatorname{Im}\left(v_{k}\right)$ becomes proportional to the absolute value $\left|d_{k}\right|$ of the amplitude $d_{k}$, according to the formula (40). Importantly, it is precisely this type of product which is proportional to the peak area $A_{k}$ in (39). In this way, the derivative envelopes in the $\mathrm{dFPT}^{(-)}$directly yield the peak areas that, in turn, are proportional to the metabolite concentrations, as stated. This is most important for overlapping resonances, such as PC and PE, which cannot even be spotted in the conventional zeroth-order derivative $(m=0)$ envelopes, let alone quantified.

\subsubsection{Noisy reconstructions}

In this sub-section, the $\mathrm{dFPT}^{(-)}$is compared with the dFFT. Both processors (Padé, Fourier) perform envelope lineshape estimations using the same noisy time signal points $\left\{c_{n}+r_{n}\right\}$ from the noise model described in Sect. 4.1.3. Here, $\left\{c_{n}\right\}$ are the noiseless FID points synthesized according to $c_{n}$ from (24). The added corruptions $\left\{r_{n}\right\}$ are a set of random numbers with zero-mean Gaussian distributions (white noise) of standard deviation $\sigma=0.0289 \mathrm{RMS}_{0}$. As stated, the presently simulated time signals are based upon FIDs encoded by in vitro MRS from breast cancer tissue [12]. Recall that the signal length on the encoded FIDs was 65536 points, as per Ref. [12]. That long signal length, which we also use for the dFFT, was needed in Ref. [12], in order to obtain reasonable appearing envelopes in the FFT. As explained, for the simulated noiseless time signal, there is no reason at all to use all the 65536 data points in the Padé-based reconstructions. The same rationale also applies to the noisy synthesized FID for which we use in the $\mathrm{FPT}^{(-)}$and $\mathrm{dFPT}^{(-)}$a much shorter signal length, $N=2048$ (the same as in Sect. 4.2.1).

The results of the reconstructions by the $\mathrm{dFPT}^{(-)}$and the dFFT are shown in Fig. 10 ( $m=3,4)$ and Fig. $11(m=5,6)$, as illustrations. No normalization is used for the $\mathrm{dFPT}^{(-)}$or the dFFT in Figs. 10 and 11. Therein, the top $(\mathrm{a}, \mathrm{b})$ and bottom $(\mathrm{c}, \mathrm{d})$ pairs of panels are for the 3rd and 4th (Fig. 10) and the 5th and 6th (Fig. 11) order derivative spectra, respectively. In Figs. 10 and 11, panels (a) and (c) are for the $\mathrm{dFPT}^{(-)}$, whereas panels (b) and (d) are for the dFFT. It is seen on Figs. 10 and 11, that on both panels (a) and (c), the $\mathrm{dFPT}^{(-)}$yields the results that are the same for the noiseless and noisy FIDs. This implies noise suppression by the 3rd-6th order differentiation transform in the $\mathrm{dFPT}^{(-)}$. On the other hand, as per panels (b) and (d) in Figs. 10 and 11, the same added noise distorts the 3rd-6th order derivative spectral envelopes in the dFFT. Even though the noise corruption level of the input FID is of a rather small standard deviation $\left(\sigma=0.0289 \mathrm{RMS}_{0}\right)$, it is remarkable that this noise is hugely amplified in 
Derivative MRS for Breast Cancer: Quantification by Shape Estimation With Synthesized Noisy FIDs Pade versus Fourier: Real Parts of Derivatives $\operatorname{Re}\left((d / d v)^{m} P_{K^{-}} / Q_{K}^{-}\right), \operatorname{Re}\left((d / d v)^{m} F_{k}\right) ; m=3,4$

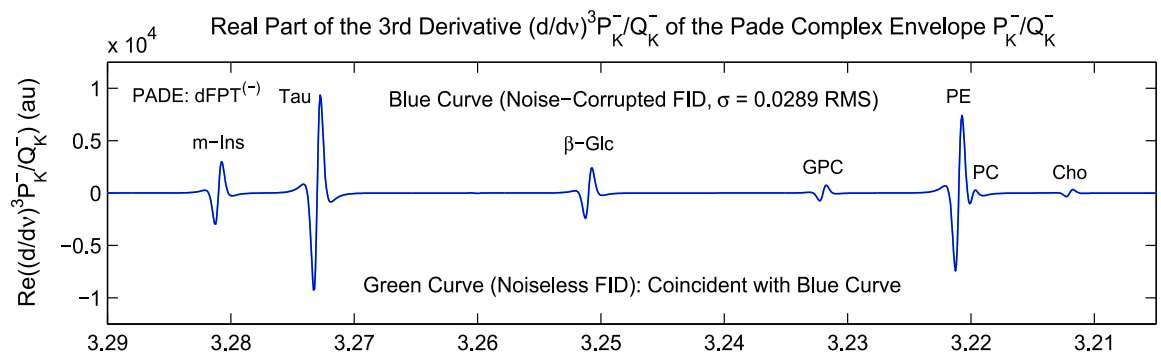

(a) Chemical Shift (ppm)

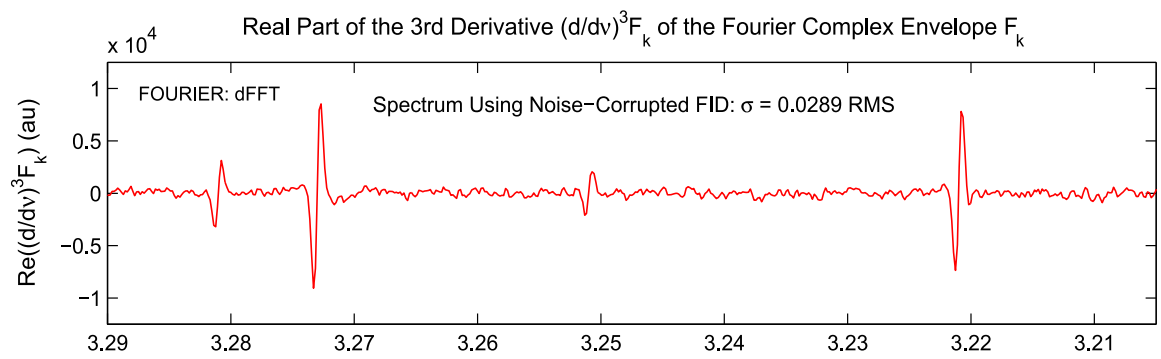

(b) Chemical Shift (ppm)

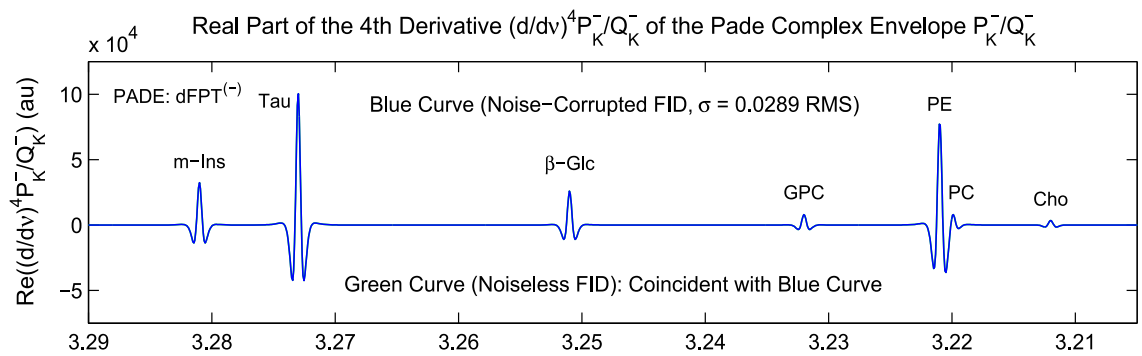

(c) Chemical Shift (ppm)

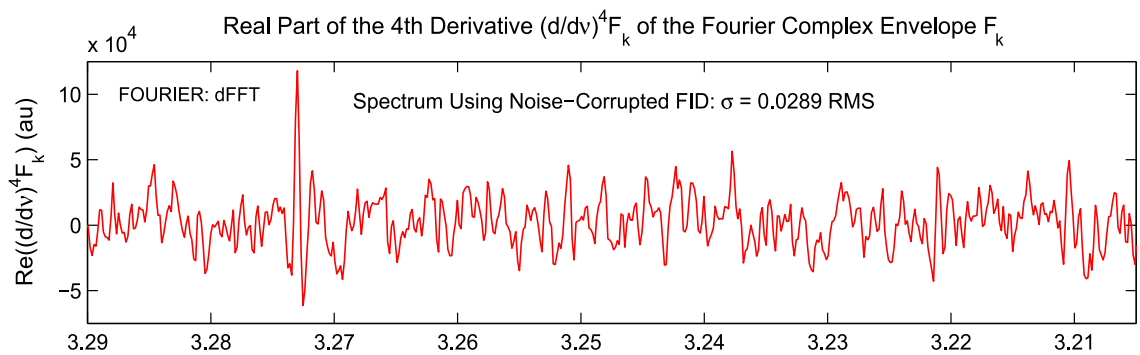

(d) Chemical Shift (ppm)

Fig. 10 The derivative fast Padé transform, $\mathrm{dFPT}^{(-)}$, versus the derivative fast Fourier transform, dFFT. Use of a synthesized noisy time signal $\left\{c_{n}+r_{n}\right\}$. The noiseless $c_{n}$ is from (24) with the input spectral parameters (37) associated with FIDs encoded by in vitro MRS from excised human breast cancer tissue, as per Ref. [12]. The addition $\left\{r_{n}\right\}$ is a set of random complex numbers obeying the Gaussian zero-mean distribution (white noise) of standard deviation $\sigma=0.0289 \mathrm{RMS}_{0}$. Real parts $\operatorname{Re}\left((\mathrm{d} / \mathrm{d} v)^{m} P_{K}^{-} / Q_{K}^{-}\right)$, $m=3(\mathbf{a}), m=4(\mathbf{c}): \mathrm{dFPT}^{(-)}$and $\operatorname{Re}\left((\mathrm{d} / \mathrm{d} v)^{m} F_{k}\right), m=3(\mathbf{b}), m=4(\mathbf{d}): \mathrm{dFFT}$. (Color online) 
Derivative MRS for Breast Cancer: Quantification by Shape Estimation With Synthesized Noisy FIDs Pade versus Fourier: Real Parts of Derivatives $\operatorname{Re}\left((\mathrm{d} / \mathrm{d} v)^{\mathrm{m}} \mathrm{P}_{\mathrm{K}}^{-} / \mathrm{Q}_{\mathrm{K}}^{-}\right), \operatorname{Re}\left((\mathrm{d} / \mathrm{d} v)^{\mathrm{m}} \mathrm{F}_{\mathrm{k}}\right) ; \mathrm{m}=5,6$

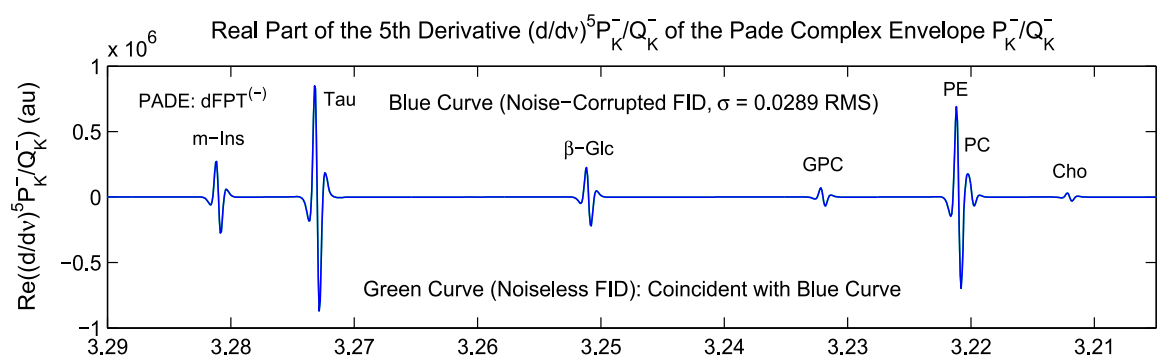

(a) Chemical Shift (ppm)
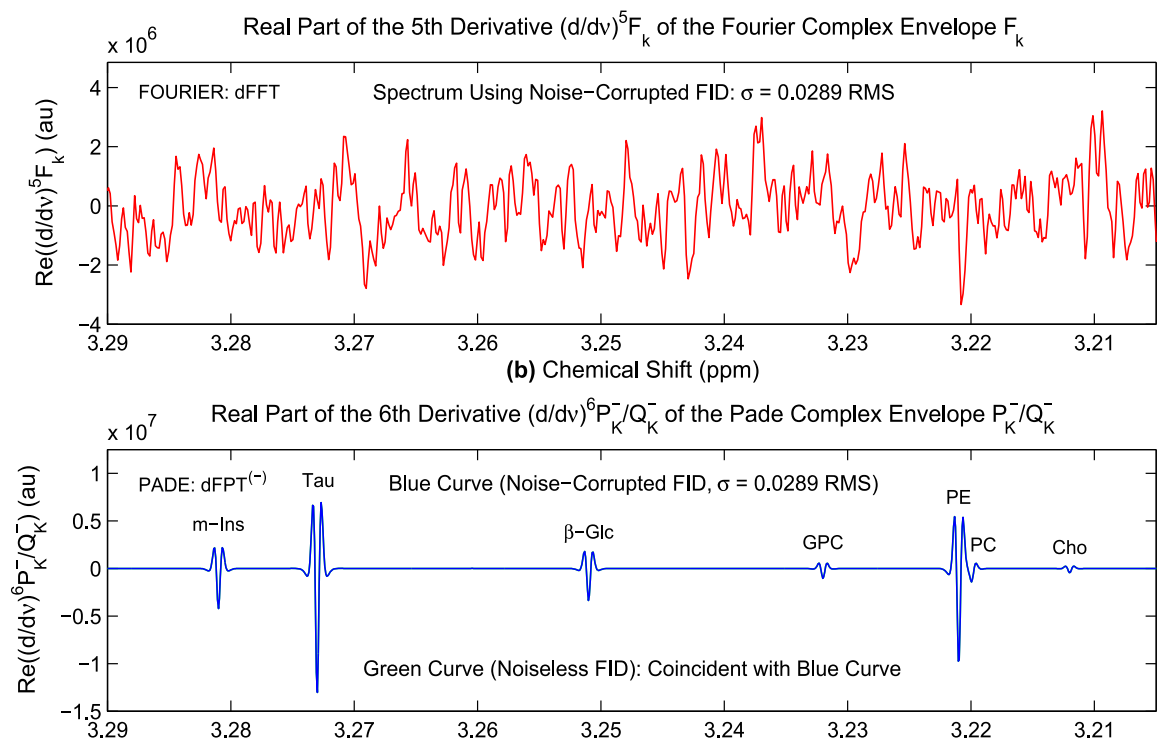

(c) Chemical Shift (ppm)

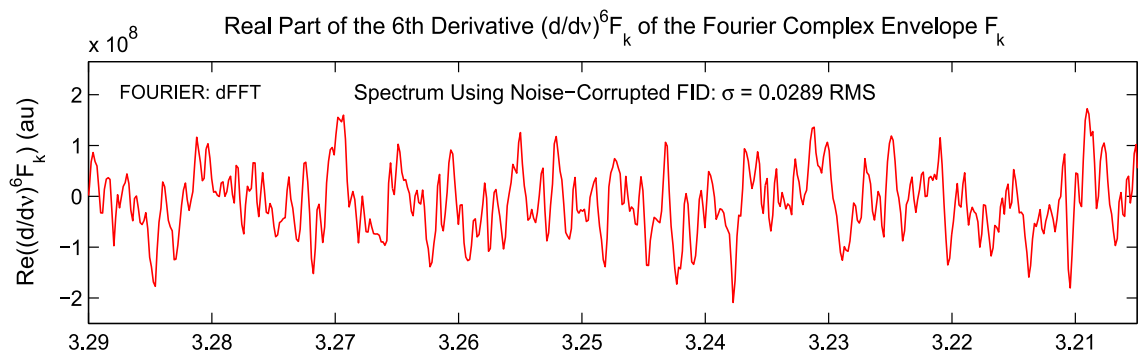

(d) Chemical Shift (ppm)

Fig. 11 The derivative fast Padé transform, $\mathrm{dFPT}^{(-)}$, versus the derivative fast Fourier transform, dFFT. Use of a synthesized noisy time signal $\left\{c_{n}+r_{n}\right\}$. The noiseless $c_{n}$ is from (24) with the input spectral parameters (37) associated with FIDs encoded by in vitro MRS from excised human breast cancer tissue, as per Ref. [12]. The addition $\left\{r_{n}\right\}$ is a set of random complex numbers obeying the Gaussian zero-mean distribution (white noise) of standard deviation $\sigma=0.0289 \mathrm{RMS}_{0}$ Real parts $\operatorname{Re}\left((\mathrm{d} / \mathrm{d} v)^{m} P_{K}^{-} / Q_{K}^{-}\right)$, $m=5(\mathbf{a}), m=6(\mathbf{c}): \mathrm{dFPT}^{(-)}$and $\operatorname{Re}\left((\mathrm{d} / \mathrm{d} v)^{m} F_{k}\right), m=5(\mathbf{b}), m=6(\mathbf{d}): \mathrm{dFFT}$. (Color online) 
the dFFT by merely proceeding from the relatively low third (Fig. 10) to the sixth-order (Fig. 11) derivatives on panels (b) and (d), respectively.

We have not shown the results in the dFFT for higher-orders $m$ of differentiation $(m \geq 7$ ) because in such cases noise completely fills the entire panels for the derivative Fourier spectra. By contrast, the results from the $\mathrm{dFPT}^{(-)}$remain the same for noisefree and noise-corrupted FIDs in the case of $m \geq 7$ (and for $0 \leq m \leq 6$ ). This demonstrates the remarkable noise suppression capability of the $m$ th differentiation operator within the Padé-based non-parametric signal processing of spectral envelopes. In sharp contrast, the derivative operator of the same order $m$ hugely amplifies noise in the Fourier-based envelopes. There is more to this juxtaposition of the non-parametric Padé vis-à-vis Fourier processing. And that is the most important bonus for noisy FIDs: the $\mathrm{dFPT}^{(-)}$can quantify, whereas the dFFT cannot, even though both estimators rely exclusively upon envelope lineshapes. When applied to noise-corrupted time signals, the dFFT yields no meaningful information, as all the physical content is buried in noise which grows with augmentation of the derivative order $m$. For example, Fig. 11 shows that in the dFFT no physical resonance can be seen on panels (b) and (d) for $m=5$ and $m=6$, respectively. Moreover, for $m=6$ in the dFFT on panel (d) the tallest noisy structure of height $\sim 1.4 \times 10^{8}$ at $3.270 \mathrm{ppm}$ surpasses by a factor of 20 the dominant double lobe of the physical resonance Tau of height $\sim 7 \times 10^{6}$ at $3.273 \mathrm{ppm}$ on panel (c) from the $\mathrm{dFPT}^{(-)}$. Note that higher order derivatives of envelopes are necessary to achieve resolution enhancement in quantification by shape estimation alone. It is these higher order derivatives (of total shape spectra) that make non-parametric Padé-based processing quantitative, in the sense of providing accurate peak positions, widths and heights for all physical resonances.

Thus, the prospect of a veritable paradigm shift is feasible. Indeed, this is a paradigm shift in all of signal processing within any spectroscopy, not only the ones based upon magnetic resonance phenomena. The reason is in the fact that despite belonging to non-parametric signal processors for qualitative lineshape estimation alone, the dFPT can split apart any number of overlapping peaks, no matter how tightly packed they might be, and exactly reconstructs their positions, widths and heights that constitute the parametric quantitative information. It should be noted that the same conclusions are drawn for the even and odd order derivative spectra. The polarity of lineshapes is opposite for even and odd derivative orders $m$ (maximae and minimae inter-change i.e. peaks become dips and vice versa). However, the steady convergence with increasing $m$ is maintained in the same way for both even and odd differentiation order. This holds true irrespective of the graphically displayed mode of spectral envelopes (real or imaginary parts, magnitudes, power).

\section{Clinical relevance of derivatives of total shape spectra through the FPT}

All the expounded characteristics of the dFPT, especially relative to the dFFT for noisy time signals, lead to the following anticipation of utmost importance, particularly for medical diagnostics by way of MRS. Being algorithmically/computationally fast, with robust noise suppression, the automatic dFPT is poised to be implemented in clinical MR scanners. With this, the long sought hope of radiologists would at last 
be realized: to visualize the entire clinical MRS information by seeing the clearly disentangled overlapping peaks and then inspecting the displayed concentrations of all the diagnostically relevant metabolites. This vision would enable the entry of MRS to the hospitals as a standard, clinically reliable part of the diagnostic armamentarium for cancer and other pathologies. The immense value of MR-based modalities in being non-invasive and free of exposure to ionizing radiation further motivates the implementation of this strategy.

\subsection{Implications for molecular imaging through MRSI}

As noted, volumetric coverage through MRSI is frequently needed for cancer diagnostics. Namely, a single voxel is often insufficiently representative of the imaged tissue. The combination of the anatomic information of MRI with the metabolic insights of MRS provides molecular imaging through MRSI. Namely, via MRSI the chemical specificity of MRS is joined with the spatial localization techniques of MRI to yield multiple MRS signals (in the time domain of encoding) over a volume of interest of tissue [4,23]. Therein, however, thousands of noisy spectra need to be processed. We can justifiably anticipate that this efficient strategy by way of derivatives of total shape spectra via the dFPT would be particularly appropriate for putting molecular imaging through MRSI into clinical practice. We would then have the derivative versions of MRS and MRSI, i.e. dMRS and dMRSI, respectively.

\subsection{Potential for improved diagnosis of a wide range of cancers}

Our motivation in choosing the problem of breast cancer is multifold. First to mention is the clinical, public health importance since breast cancer is the most frequently occurring malignancy and cause of cancer-related deaths among women across the world [24], together with the well-established value of early breast cancer detection for improved survival [24-26]. Further improvements in diagnostic accuracy over the anatomic imaging methods via MRI are anticipated by molecular imaging through MRS and MRSI [27]. However, as reviewed in detail in Ref. [2], reliance upon Fourierbased processing of the encoded FIDs has severely limited the potential added value of MRS and MRSI for improving the accuracy with which breast cancer is detected and distinguished from benign pathology. In the present study, on the Padé-based lineshape estimations, not only do we succeed in identifying the breast cancer biomarker, phosphocholine, PC, by non-parametric analysis alone, but for the first time through the derivative spectra in the dFPT, exact quantification is also achieved through this efficient strategy.

It should be emphasized that PC is also a biomarker of other cancers [28-30]. This may be related to loss of tumor suppressor p53 function [31]. Thus far, it has only been through parametric analysis with the FPT, that PC has been identified via in vivo encoded FIDs from proton MRS from brain and ovarian tumors [32,33]. Further, it should be underscored that dense spectra with many other overlapping resonances of diagnostic importance are also characteristic of brain tumors, ovarian cancers as well as prostate, lung and other cancers [19,22,32-34]. We have pointed out that 
there is a major potential for synergistic development, such that each step forward with Padé-optimized MRS in cancer diagnostics, informs and advances other areas [22]. This statement now becomes particularly salient, with the possibilities offered by non-parametric quantification using derivative spectra in the dFPT, in practical implementation of in vivo MRS and MRSI for early cancer diagnostics, as well as for other aspects of cancer care.

\section{Conclusions and perspectives}

It is well-known that total shape spectra or envelopes have previously provided only partial, qualitative estimations on the processed time signals. Such extracted information is qualitative because it exclusively concerns the lineshapes of envelopes. It is partial, since the spectral content hidden within the usually abundant compound peaks tightly packed with overlapping resonances in envelopes has remained generally inaccessible.

Conventionally, all non-parametric signal processors are limited to shape estimations alone. For reconstruction of the fundamental eigen-pairs (frequencies and amplitudes, both complex), parametric estimation is needed to solve the inverse problem called "spectral analysis" or quantification.

The parametric FPT is a prime example of a signal processor capable of exactly reconstructing all the genuine frequencies and amplitudes from synthesized (noisefree, noise-corrupted) and encoded time signals. The complex frequencies are themselves a pair of real numbers representing the chemical shifts (location) and widths of resonances or peaks. Complex amplitudes are also a tandem of values: the amplitude magnitudes (absolute value of the complex amplitudes) and the amplitude phases. Thus, the parametric FPT provides four real parameters per resonance: the peak position, the peak width, as well as the magnitude and phase of the amplitude. On theoretical grounds, all the amplitude phases are expected to be zero-valued in time signals encoded by magnetic resonance spectroscopy, MRS, which is the main focus of the present study. For the reason of various imperfections in encoding, the phases of time signal amplitudes are non-zero, and the so-called zeroth- and 1st-order phase corrections are usually made to produce partially positive-definite absorptive envelopes.

Alternatively, and by definition, spectral envelopes are always strictly positivedefinite in their magnitude modes (absolute values). This has not been used in practice within MRS. The reasons are the peak width broadening in customary (non-derivative) magnitude envelopes, and their distortions by the interference (destructive, constructive) of the constituent absorptions and dispersions. Moreover, widening of the bottom of peaks in the magnitude modes of non-derivative envelopes, makes weaker resonances (near the background level) even more hidden beneath the long tails of lineshapes than in the associated absorptive envelopes. If not for these obstacles, the magnitude mode would be most convenient, because it does not necessitate any phase corrections (that are subjective and arbitrary), and yet it provides positive-definite envelopes from the onset. 
Fortunately, however, the mentioned disadvantages of the envelope magnitudes are restricted only to the conventional, non-derivative total shape spectra. For the derivative envelopes in the magnitude mode, the universal pattern is diametrically opposite. Namely, with augmentation of the differentiation order $m$ in the magnitude modes of the envelopes in the derivative fast Padé transform, dFPT, via $\left|(\mathrm{d} / \mathrm{d} v)^{m} P_{K} / Q_{K}\right|$, the peak widths gradually decrease and the resonance tails flatten to the baseline level of the background. This enables the dFPT to separate the overlapped peaks in compound spectral structures and to make the hidden peaks, even the weakest, emerge in an unequivocal manner.

The dFPT does not stop at visualization of the hidden resonances. It proceeds with a critical step further by exactly quantifying all the reconstructed physical resonances, isolated and overlapped alike, despite performing shape estimation alone. Most importantly, as opposed to the derivative fast Fourier transform, dFFT, where differentiation hugely amplifies noise, the dFPT acts as an efficient noise suppressor, i.e. a noise filter. All these features are presently illustrated for synthesized noiseless and noisy time signals reminiscent of the corresponding data encoded by in vitro MRS from excised breast cancer tissue. In breast tumor diagnostics via MRS, a key achievement would be to reliably identify and quantify the recognized breast cancer biomarker, phosphocholine, PC. This is one of the most demanding tasks for any non-parametric signal processing method including the non-derivative FPT, since the PC resonance is completely invisible in customary zeroth-order $(m=0)$ derivative envelopes due to the dominant adjacent resonance of phosphoethanolamine, PE. The PC-to-PE chemical shift separation is only $0.001 \mathrm{ppm}$.

We show, however, that the dFPT successfully solves this problem by unambiguously identifying and quantifying all the genuine resonances, including the PC peak. In this way, with the dFPT, one would have on the same screen not only visualized the PC-PE separation, but also the automatically, objectively and reliably reconstructed concentrations of these two metabolites, alongside the corresponding numerical parameters for all the other molecules. The same opportunities apply to any other signal processing problem that poses diagnostic dilemmas in MRS for oncology and beyond. More broadly, the dFPT is anticipated to find useful applications in other areas of both research and technology, especially when signal processing is faced with the usually insurmountable problem of crowded spectra laden with unresolved resonances that could contain vitally important information.

Overall, it can be patently concluded that the higher-order differentiation transform, when used in conjunction with the derivative fast Padé transform, dFPT, simultaneously achieves resolution enhancement, noise suppression and exact quantification, despite explicit non-parametric processing of envelope lineshapes alone (i.e. without solving the quantification problem).

Over the years, firmly grounded was the parametric version of the FPT which performs explicit quantification by finding the exact solution of the problem of spectral analysis for the fundamental complex frequencies and the corresponding complex amplitudes. This variant of the FPT, quantification-equipped from the onset, as the gold standard, continues to be irreplaceable and, as such, should always be applied to any time signal, synthesized or encoded alike. The non-parametric version of the FPT, enriched by the differentiation transform, yields the derivative fast Padé transform, 
dFPT. The standard non-parametric FPT (the zeroth-order derivative $m=0$ ) is not for quantification, since it is restricted to estimation of spectral envelope lineshapes. However, the dFPT is quantification equipped despite being a non-parametric shape estimator. It is the differential transforms, especially of higher orders of derivatives, that are responsible for establishing the quantification capabilities of an initially designed non-parametric shape estimator. In other words, the dFPT acquires quantification features en route, by differentiation. As such, the non-parametric quantification-equipped dFPT is not meant to be a substitute for its parametric quantification-equipped FPT. Rather, the non-parametric dFPT and parametric FPT are complementary, and should be applied in concert to the same input time signals, so as to cross-validate each other. Crucially, in very different ways, both the parametric FPT and the non-parametric dFPT reconstruct all the component spectra of every physical resonance in the studied envelope.

The non-parametric dFPT could, for example, be used first to quickly display the gestalt right on the screen: the spectral lineshapes and the parameter linelist (peak position, widths, heights, and component concentrations) provided by the higher-order derivatives. This first insight should be subsequently checked for corroboration with the direct solution of the quantification problem by the parametric FPT. It is in this way that these two versions of the fast Padé transform (derivative dFPT and non-derivative FPT) should run hand-in-hand to reconstruct the most reliable information from the input time signals. Such a perspective is crucially important for interdisciplinary applications. For MRS in medicine, this avenue has special ramifications, as it is poised to provide the long-sought clinical reliability particularly for early cancer diagnostics.

Acknowledgements This work was supported by King Gustav the 5th Jubilee Fund, The Marsha Rivkin Center for Ovarian Cancer Research and FoUU through Stockholm County Council to which the authors are grateful.

Open Access This article is distributed under the terms of the Creative Commons Attribution 4.0 International License (http://creativecommons.org/licenses/by/4.0/), which permits unrestricted use, distribution, and reproduction in any medium, provided you give appropriate credit to the original author(s) and the source, provide a link to the Creative Commons license, and indicate if changes were made.

\section{References}

1. Dž. Belkić, Quantum-Mechanical Signal Processing and Spectral Analysis (Institute of Physics Publishing, Bristol, 2005)

2. Dž. Belkić, K. Belkić, Visualizing hidden components of envelopes non-parametrically in magnetic resonance spectroscopy: phosphocholine, a breast cancer biomarker. J. Math. Chem 55, 1698-1723 (2017)

3. Dž. Belkić, K. Belkić, Robust identification of the cancer biomarker phosphocholine through partitioned envelopes in noisy magnetic resonance spectroscopic data by the non-parametric fast Padé transform. J. Math. Chem 55, 2004-2047 (2017)

4. R.W. Prost, Magnetic resonance spectroscopic imaging, in Magnetic Resonance Imaging and Spectroscopy, Volume 3, Comprehensive Biomedical Physics, ed. by Dž. Belkić, K. Belkić (Elsevier, Amsterdam, 2014), pp. 331-345

5. Dž. Belkić, Fast Padé transform (FPT) for magnetic resonance imaging and computerized tomography. Nucl. Instrum. Methods Phys. Res. A 471, 165-169 (2001)

6. W.H. Press, S.A. Teukolsky, W.T. Vetterling, B.P. Flannery, Numerical Recipes in Fortran 77: The Art of Scientific Computing, 2nd edn. (Cambridge University Press, Cambridge, 1992) 
7. R. Courant, D. Hilbert, Methods of Mathematical Physics (Interscience, New York, 1953)

8. Dž. Belkić, K. Belkić, The general concept of signal-noise separation (SNS): mathematical aspects and implementation in magnetic resonance spectroscopy. J. Math. Chem. 45, 563-597 (2009)

9. E.T. Bell, Exponential polynomials. Ann. Math. 35, 258-277 (1934)

10. R.E. Dinnebier, S.J.L. Billinge, Powder Diffraction: Theory and Practice (The Royal Society of Chemistry Publishing, Cambridge, 2008)

11. P. van der Heide, X-Ray Photoelectron Spectroscopy: An Introduction of Principles and Practices (Wiley, Hoboken, 2012)

12. I.S. Gribbestad, B. Sitter, S. Lundgren, J. Krane, D. Axelson, Metabolite composition in breast tumors examined by proton nuclear magnetic resonance spectroscopy. Anticancer Res. 19, 1737-1746 (1999)

13. Dž. Belkić, Strikingly stable convergence of the Fast Padé transform (FPT) for high-resolution parametric and non-parametric signal processing of Lorentzian and non-Lorentzian spectra. Nucl. Instrum. Methods Phys. Res. A 525, 366-371 (2004)

14. Dž. Belkić, K. Belkić, The fast Padé transform in magnetic resonance spectroscopy for potential improvements in early cancer diagnostics. Phys. Med. Biol. 50, 4385-4408 (2005)

15. Dž. Belkić, K. Belkić, In vivo magnetic resonance spectroscopy by the fast Padé transform. Phys. Med. Biol. 51, 1049-1075 (2006)

16. Dž. Belkić, K. Belkić, Mathematical modeling applied to an NMR problem in ovarian cancer detection. J. Math. Chem. 43, 395-425 (2008)

17. Dž. Belkić, K. Belkić, Signal Processing in Magnetic Resonance Spectroscopy with Biomedical Applications (Taylor \& Francis Publishers, London, 2010)

18. Dž. Belkić, K. Belkić, Resolution enhancement as a key step towards clinical implementation of Padéoptimized magnetic resonance spectroscopy for diagnostic oncology. J. Math. Chem 51, 2608-2637 (2013)

19. Dž. Belkić, K. Belkić, The fast Padé transform for noisy magnetic resonance spectroscopic data from the prostate: potential contribution to individualized prostate cancer care. J. Math. Chem. 54, 707-764 (2016)

20. Dž. Belkić, K. Belkić, Proof-of-the-concept study on mathematically optimized magnetic resonance spectroscopy for breast cancer diagnostics. Technol. Cancer Res. Treat. 14, 277-297 (2015)

21. Dž. Belkić, K. Belkić, Mathematically-optimized magnetic resonance spectroscopy in breast cancer diagnostics: implications for personalized cancer medicine. J. Math. Chem. 54, 186-230 (2016)

22. Dž. Belkić, K. Belkić, Robust high-resolution quantification of time signals encoded by in vivo magnetic resonance spectroscopy. Nucl. Instrum. Methods Phys. Res. A (2017). https://doi.org/10.1016/j.nima. 2017.07.034

23. S.J. Nelson, E. Graves, A. Pirzkall, X. Li, A. Antiniw Chan, D.B. Vigneron, T.R. McKnight, In vivo molecular imaging for planning radiation therapy of gliomas: an application of $1 \mathrm{H}$ MRSI. J. Magn. Reson. Imaging 16, 464-476 (2002)

24. R.A. Smith, K.S. Andrews, D. Brooks, S.A. Fedewa, D. Manassaram-Baptiste, D. Saslow, O.W. Brawley, R.C. Wender, Cancer screening in the United States, 2017: a review of current American Cancer Society guidelines and current issues in cancer screening. CA Cancer J. Clin. 67, 100-121 (2017)

25. L. Pace, N. Keating, A systematic assessment of benefits and risks to guide breast cancer screening decisions. J. Am. Med. Assoc. 311, 1327-1335 (2014)

26. S. Njor, W. Schwartz, M. Blicert-Toft, E. Lynge, Decline in breast cancer mortality: how much is attributable to screening? J. Med. Screen. 22, 20-27 (2015)

27. M.F. Kircher, H. Hricak, S.M. Larson, Molecular imaging for personalized cancer care. Mol. Oncol. 6, 182-195 (2012)

28. K. Glunde, J. Jiang, S.A. Moestue, I.S. Gribbestad, MRS/MRSI guidance in molecular medicine: targeting choline and glucose metabolism. NMR Biomed. 24, 673-690 (2011)

29. E. Iorio, D. Mezzanzanica, P. Alberti, F. Spadaro, C. Ramoni, S. D’Ascenzo, D. Millimaggi, A. Pavan, V. Dolo, S. Canavari, F. Podo, Alterations of choline phospholipid metabolism in ovarian tumor progression. Cancer Res. 65, 9369-9376 (2005)

30. N.P. Davies, M. Wilson, L.M. Harris, K. Natarajan, S. Lateef, L. MacPherson, S. Sgouros, R.G. Grundy, T. Arvanitis, A. Peet, Identification and characterization of childhood cerebellar tumors by in vivo proton MRS. NMR Biomed. 21, 908-918 (2008)

31. N. Mori, R. Delsite, K. Natarajan, M. Kulawiec, Z. Bhujwalla, K. Singh, Loss of p53 function in colon cancer cells results in increased phosphocholine and total choline. Mol. Imaging 3, 319-323 (2004) 
32. Dž. Belkić, K. Belkić, Improving the diagnostic yield of magnetic resonance spectroscopy for pediatric brain tumors through mathematical optimization. J. Math. Chem 54, 1461-1513 (2016)

33. Dž. Belkić, K. Belkić, In vivo magnetic resonance spectroscopy for ovarian cancer diagnostics. J. Math. Chem. 55, 349-405 (2017)

34. W. Chen, S. Lu, J. Ou, G. Wang, Y. Zu, F. Chen, C. Bai, Metabonomic characteristics and biomarker research of human lung cancer tissues by HR 1H NMR spectroscopy. Cancer Biomarkers 16, 653-664 (2016) 